\title{
The value of single-molecule real-time technology in the diagnosis of rare thalassemia variants and analysis of phenotype-genotype correlation
}

\author{
Shiqiang Luo ${ }^{1,2,7}$, Xingyuan Chen ${ }^{3,4,7}$, Dingyuan Zeng ${ }^{5}$, Ning Tang ${ }^{5}$, Dejian Yuan ${ }^{1,2}$, Qingyan Zhong ${ }^{1,2}$, Aiping Mao ${ }^{6}$, Ruofan Xu ${ }^{6}$ and \\ Tizhen $\operatorname{Yan}^{1,2 凶}$
}

(C) The Author(s) 2021

To compare single-molecule real-time technology (SMRT) and conventional genetic diagnostic technology of rare types of thalassemia mutations, and to analyze the molecular characteristics and phenotypes of rare thalassemia gene variants, we used 434 cases with positive hematology screening as the cohort, then used SMRT technology and conventional gene diagnosis technology [(Gap-PCR, multiple ligation probe amplification technology (MLPA), PCR-reverse dot blot (RDB)] for thalassemia gene screening. Among the 434 enrolled cases, conventional technology identified 318 patients with variants (73.27\%) and 116 patients without variants (26.73\%), SMRT identified 361 patients with variants (83.18\%), and 73 patients without variants (16.82\%). The positive detection rate of SMRT was $9.91 \%$ higher than conventional technology. Combination of the two methods identified 485 positive alleles among 49 types of variant. The genotypes of 354 cases were concordant between the two methods, while 80 cases were discordant. Among the 80 cases, 76 cases had variants only identified in SMRT method, 3 cases had variants only identified in conventional method, and 1 false positive result by the traditional PCR detection technology. Except the three variants in HS40 and HBG1-HBG2 loci, which was beyond the design of SMRT method in this study, all the other discordant variants identified by SMRT were validated by further Sanger sequencing or MLPA. The hematological phenotypic parameters of 80 discordant cases were also analyzed. SMRT technology increased the positive detection rate of thalassemia genes, and detected rare thalassemia cases with variable phenotypes, which had great significance for clinical thalassemia gene screening.

Keywords: Single molecule real-time technology (SMRT); $a$-thalassemia rare variant; $\beta$-thalassemia rare variant; Carrier screening Journal of Human Genetics (2022) 67:183-195; https://doi.org/10.1038/s10038-021-00983-1

\section{INTRODUCTION}

Thalassemia, also known as Mediterranean anemia, is a hereditary hemolytic anemia mainly caused by deletions or point mutations of globin genes. It is one of the most common single gene diseases in the world. The global thalassemia gene carriers comprise $\sim 1.67 \%$ of the total population, which are mainly distributed in the Mediterranean coast, North Africa, the Middle East, the Indian mainland, Southeast Asia, and southern China [1]. Thalassemia is one of the most common genetic diseases in southern China. The pathogenic variants of thalassemia include single-nucleotide variations (SNVs), indels, and large fragments of copy number variants (CNVs) and structural variations (SVs). Among them, a-thalassemia is mainly caused by large fragment deletions, and $\beta$-thalassemia mainly involves point mutations. In China, simple and low cost red blood cell and hemoglobin tests are used as a first-tier screening strategy. Then molecular diagnosis will be performed for individuals with positive results of blood test. Conventional molecular diagnosis methods for detecting thalassemia genes include Gap-PCR, PCR-RDB, PCR-flow fluorescence hybridization, and MLPA. Other common technologies used in China include gene chip, Sanger sequencing, and next generation sequencing (NGS). Conventional screening methods can only detect a limited spectrum of gene mutations, which sometimes lead to misdiagnosis. NGS used in thalassemia screening can effectively reduce the need for various types of conventional genetic testing, but there could be missed diagnoses [2]. Although the probe hybridization target capture NGS method can simultaneously detect deletions and SNV/indels, the detection cost is high, and the accuracy is not ideal. Gap-PCR combined with NGS technology is currently used to compensate for the shortcomings of NGS capture sequencing technology. In addition, due to the high homology between $H B A 2$ and $H B A 1$ genes, the short-

\footnotetext{
'Department of Medical Genetics, Liuzhou Key Laboratory of Birth Defects Prevention and Control, Liuzhou Maternity and Child Healthcare Hospital, 545001 Liuzhou, Guangxi, China. 'Liuzhou Key Laboratory of Reproductive Medicine, 545001 Liuzhou, Guangxi, China. ${ }^{3}$ Department of Laboratory Medicine, The People's Hospital of Guangxi Zhuang Autonomous Region, Nanning, Guangxi Zhuang Autonomous Region, China. ${ }^{4}$ Guangxi Academy of Medical Sciences, Nanning, Guangxi Zhuang Autonomous Region, China. ${ }^{5}$ Guangxi Health Commission Key Laboratory of Birth Cohort Study in Pregnant Women of Advanced Age, 545001 Liuzhou, Guangxi, China. ${ }^{6}$ Berry Genomics Corporation, 102200 Beijing, China. ${ }^{7}$ These authors contributed equally: Shiqiang Luo, Xingyuan Chen. ${ }^{凶}$ email: luobu_0971@163.com
}

Received: 29 July 2021 Revised: 19 September 2021 Accepted: 29 September 2021

Published online: 25 October 2021 
read NGS method cannot distinguish $H B A 2$ and $H B A 1$ effectively $[3,4]$. With the advantage of long-molecule sequencing, PacBio real-time sequencing technology (SMRT) had been used for comprehensive and precious thalassemia test $[5,6]$. In this study, SMRT technology and conventional methods were performed for 434 suspected carriers of thalassemia to simultaneously detect deletion and non-deletion variants of a-thalassemia and $\beta$-thalassemia. Compared to conventional methods, SMRT technology detected more abnormal hemoglobin variant sites on the $H B A 1, H B A 2$, and $H B B$ genes, which illustrated the value of SMRT technology in the diagnosis of common and rare types of $a-$ thalassemia and $\beta$-thalassemia variants.

\section{PATIENTS AND METHODS \\ Patients}

A total of 434 patients who attended Liuzhou Maternal and Child Health Hospital in Guangxi, China from January 2018 to December 2020, were included in the study. The enrolled patients should meet at least one of the following inclusion criteria: (1) routine hematology examination showed abnormal mean corpuscular volume $(\mathrm{MCV} \leq 80 \mathrm{fL})$ and/or mean corpuscular hemoglobin $(\mathrm{MCH} \leq 27 \mathrm{pg})$; (2) hemoglobin electrophoresis showed $\mathrm{HbA} 2<2.5 \%$ or $\mathrm{HbA} 2 \geq 3.5 \%$ or elevated $\mathrm{HbF}$ or abnormal hemoglobin; (3) the results of conventional genetic diagnosis were inconsistent with the results of the hematology phenotype; (4) the patient gave birth to children with moderate or severe thalassemia; and (5) there may be abnormalities outside the scope of conventional genetic testing techniques. The exclusion criteria included: (1) incomplete basic clinical data; (2) the patient had other blood diseases; and (3) the patient had mental abnormalities or cognitive dysfunctions. The study group was comprised of 185 males and 249 females, with age range 3 days to 56 years, and an average age of $26.4 \pm 12.59$ years. This study was approval by the ethics committee of our hospital, and all research subjects or their legal guardians signed an informed consent form.

\section{Methods}

Hematology and hemoglobin electrophoresis analysis. An automatic blood cell analyzer was used for routine blood analyses, and high-performance liquid chromatography was used for hemoglobin analysis to detect $\mathrm{HbF}$, $\mathrm{HbA2}, \mathrm{HbH}$, and other hemoglobin variants.

Genomic DNA extraction. The magnetic bead method was used to extract nucleic acids (LabAid820; Xiamen Zhishan Biotechnology, Xiamen, China). The nucleic acid analyzer (ASP-2680; ACTGene, Piscataway, NJ, USA) was used to detect DNA concentration and purity. The $A_{260} / A_{280}$ of extracted DNA was between 1.6 and 1.9 , and the concentration was $20-30 \mathrm{ng} / \mu \mathrm{L}$.

$a$-thalassemia and $\beta$-thalassemia genotyping. Genomic DNA extracted from peripheral blood were used for thalassemia test. Gap-PCR (Yishengtang, Shenzhen, China) was performed for the four common a-thalassemia deletions [-- ${ }^{\text {SEA }}$ (Southeast Asia), $-a^{3.7}$ (rightward), $-a^{4.2}$ (leftward) -- THAI (Thailand)] were performed using the gap-polymerase chain reaction (GapPCR). PCR-RDB assay (Yishengtang, Shenzhen, China) was performed for the three common non-deletional a-thalassemia mutations including $\mathrm{Hb}$ Constant Spring ( $\mathrm{Hb} \mathrm{CS}, H B A 2$ : c.427T>C), Hb Quong Sze ( $\mathrm{Hb} \mathrm{QS}, \mathrm{HBA2}$ : c.377T $>C$ ), and $\mathrm{Hb}$ Westmead (Hb WS, HBA2: $\mathrm{C.369G}>\mathrm{C}$ ), and the 17 known $\beta$-thalassemia mutations including $-28(A>G)(H B B: C .-76 A>G),-29(A>G)$ (HBB: c. $-79 A>G),-30(T>C)(H B B:$ c. $-80 T>C),-32(C>A)(H B B: C .-82 C>A)$, codons 14/15 (+G) (HBB: c.45_46insG), codon 17 (A>T) (HBB: c.52A>T), codon 26 (or $\mathrm{Hb}$ E) $(\mathrm{G}>\mathrm{A})(\mathrm{HBB}: \mathrm{c} .79 \mathrm{G}>\mathrm{A})$, codons $27 / 28(+\mathrm{C})(\mathrm{HBB}: \mathrm{c} .84$ 85insC), codon 31 (-C) (HBB: c.94delC), codons 41/42 (-TTCT) (HBB: c.126_129delCTT), codon $43(\mathrm{G}>\mathrm{T})(H B B: \mathrm{c} .130 \mathrm{G}>\mathrm{T})$, codons 71/72 (+A) (HBB: c. 216_217insA), IVS-I-1 (G>T) (HBB: c.92+1G>T), IVS-I-5 (G>C) (HBB: c.92+5G>C), IVS-II-654 (C>T) (HBB: c.316-197C >T), CAP+1 (A>C) (HBB: c. $-50 A>C)$, and initiation codon $(\mathrm{T}>\mathrm{G})$ (HBB:c.2T>G). MLPA detection was performed using the P102 and P140 probe kit (MRC-Holland, Amsterdam, The Netherlands) to analyze the copy number variation of the deleted plutonium-Mediterranean gene. Capillary electrophoresis was performed on amplified products using the 3500Dx genetic Analyzer (Applied Biosystems, Foster City, CA, USA).

SMRT and data analysis. Genomic DNA was extracted from peripheral blood leukocytes using the QIAamp DNA blood mini kit (Qiagen, Hilden,

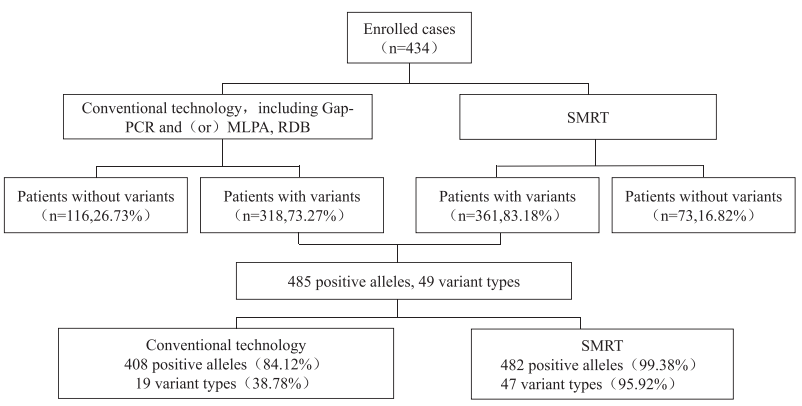

Fig. 1 Combined analyses of the two techniques. Comparison of variants detection results between conventional technology and SMRT technology.

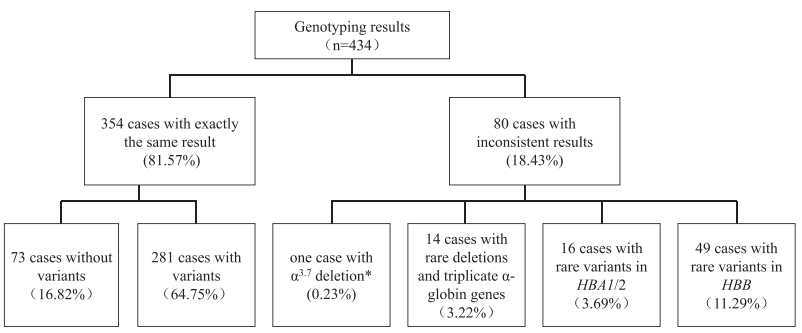

Fig. 2 Comparison of genotyping results between conventional technology and SMRT technology *SMRT method showed that the genotype of sample D141966 was $-\alpha^{3.7} / \alpha \alpha$, while by conventional Gap-PCR it was $-\alpha^{3.7} /-\alpha^{3.7}$. Validation by MLPA confirmed D141966 had heterozygous $-\alpha^{3.7}$ deletion.

Germany). Purified DNA samples were quantified using the Qubit dsDNA BR assay kit (Thermo Fisher Scientific, Waltham, MA, USA) using a Qubit 2.0 fluorometer (Life Technologies, Carlsbad, CA, USA). Samples were sent to an independent laboratory (Berry Genomics, Beijing, China) for sequencing using the Sequel II platform and data analysis (PacBio, Menlo Park, CA, USA). Briefly, genomic DNA samples were subjected to multiplex longmolecule PCR using optimized primers to generate specific amplicons that encapsulated currently known structural variation (SV) regions, singlenucleotide variations (SNVs), and indels (insertions and deletions) in the $H B A 1, H B A 2$, and $H B B$ genes. After purification and end repair, the barcoded adapters were ligated to the $5^{\prime}$ and $3^{\prime}$ ends, and SMRT bell libraries were prepared using the sequel binding and internal Ctrl Kit 3.0 (PacBio). Primed DNA-polymerase complexes were loaded onto SMRT cells (PacBio) and sequencing was performed on the PacBio Sequel II system to generate 10-25 subjects per molecule. Following alignment of subreads, the consensus circular sequence was mapped to the GRCh38 reference and variants identified (FreeBayes software, version 1.2.0). Variant pathogenicity was classified according to general guidelines and from information provided in hemoglobin variant databases. Phenotypes were finally assigned from known genotypic-phenotypic associations. Large deletion variants was confirmed by Gap-PCR or MLPA. SNVs and indels were confirmed by PCR-RDB or Sanger sequencing.

\section{Sanger sequencing for $H B A$ and $H B B$ gene}

Four sets of primer pairs were designed and used to amplify and sequence the a-globin genes (HBA1 and HBA2) and $\beta$-globin gene (HBB): HBA1-F: $5^{\prime}-$ TGG AGG GTG GAG ACG TCC TG-3'; HBA1-R: 5'-TCC ATC CTC TCC TCC CGC CCC TGC CTT TTC-3'; HBA2-F: 5'-TGG AGG GTG GAG ACG TCT TG-3'; HBA2-R: 5'-CCG TTG TTG GCA CAT TCC GG-3';HBB-FP: 5'-AAC TCC TAA GCC AGT GCC AG-3'; Avall-HBB-FP: $5^{\prime}-T$ TG GGG ATC TGT CCA CTC CT-3'; Avall-HBB-RP: $5^{\prime}-$ CCA GCC TTA TCC CAA CCA TAA AAT AA-3'; and HBB-RP: 5'-ATG CAC TGA CCT CCC ACA TTC CCT-3'. DNA sequencing was performed using the Sanger dideoxy termination sequencing method, and the reference sequence was NM_000517. Amplification was performed using $50 \mathrm{ng}$ of genomic DNA, and 20 pmol of forward $(F)$ and reverse (R) primers, on a C1000 Thermal Cycler (Bio-Rad Laboratories, Hercules, CA, USA). The PCR products were sequenced on the ABI PRISM ${ }^{\circ} 3130$ automated sequencer (Applied Biosystems). 


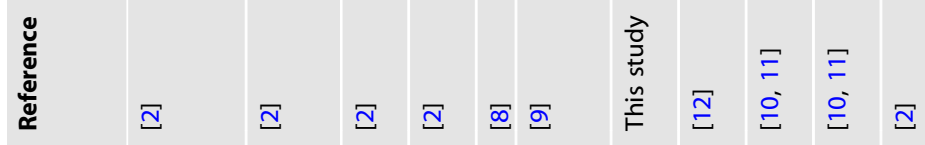

$\Xi \Xi \stackrel{\bar{m}}{\Xi} \stackrel{\Xi}{\Xi}$

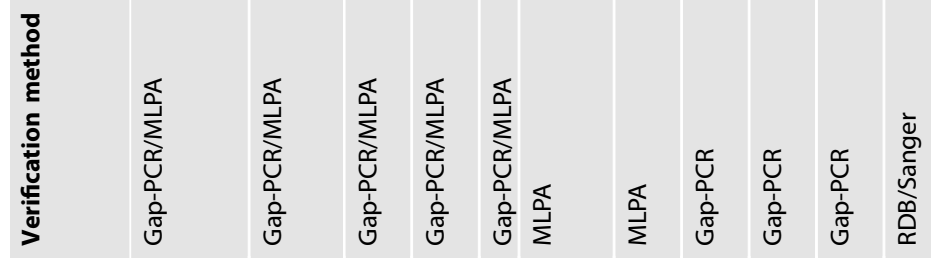

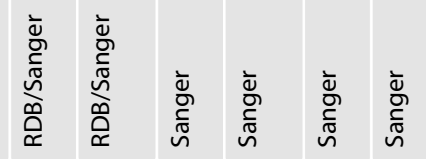

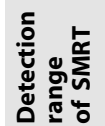

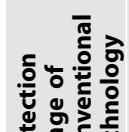

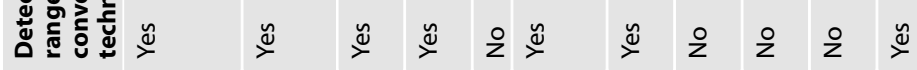

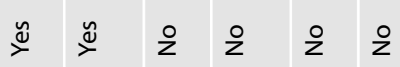

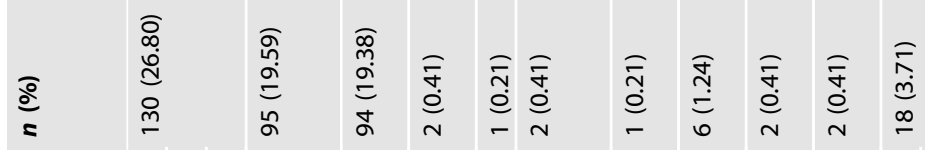

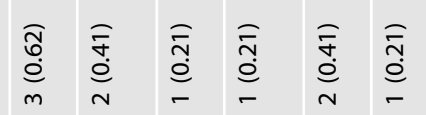

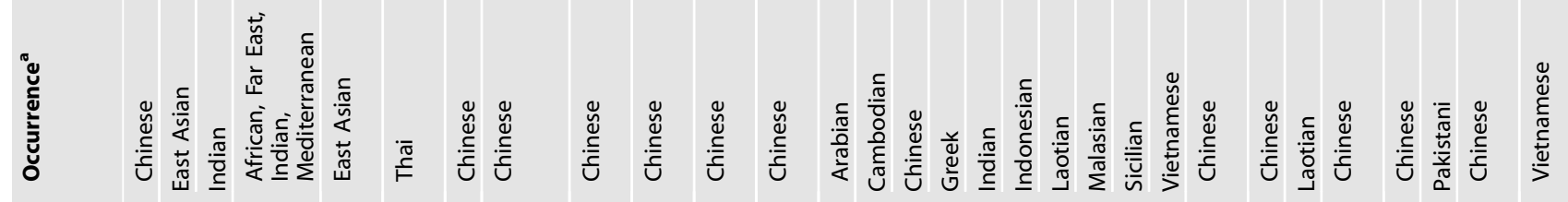

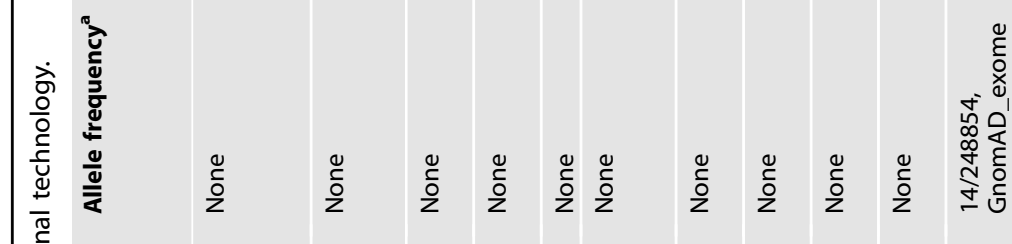

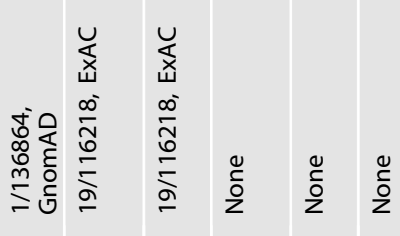

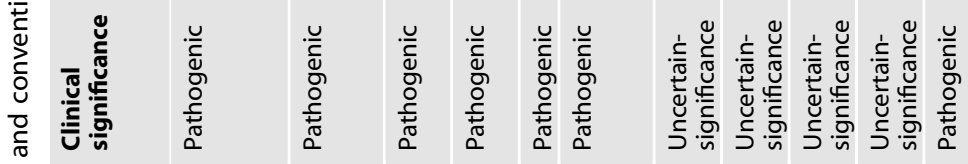

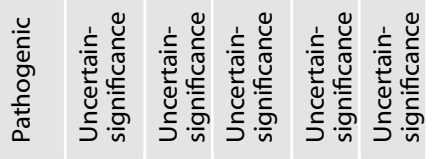

i

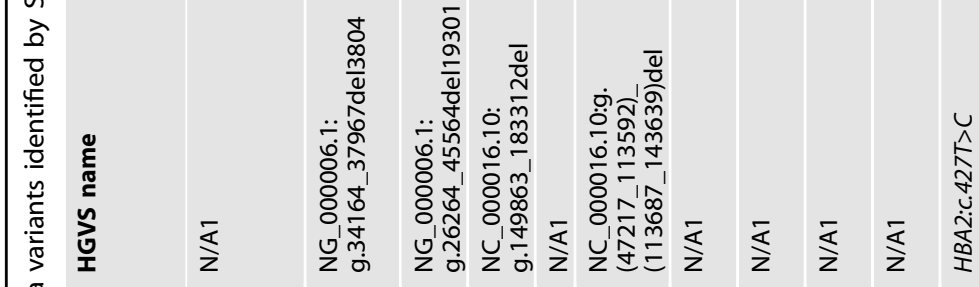

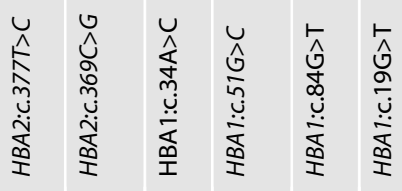

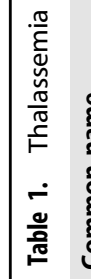

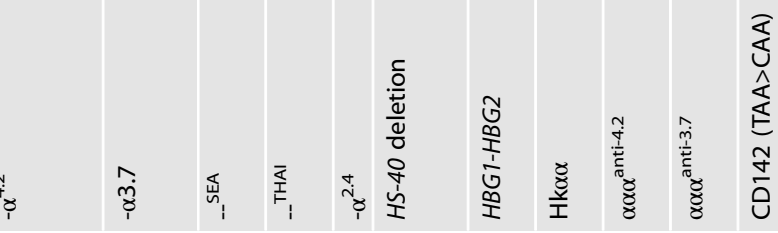

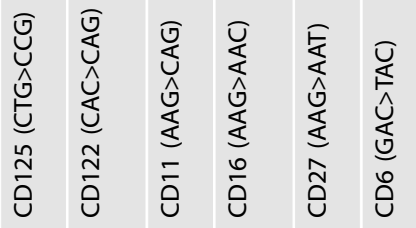




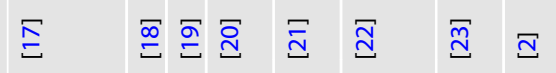

:

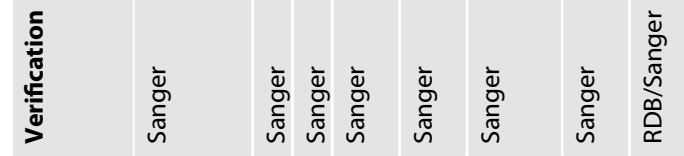

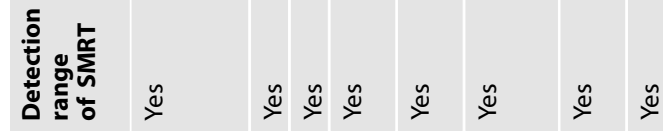

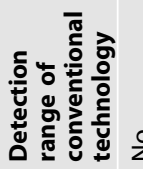

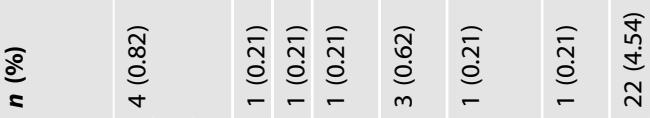

ㄱ

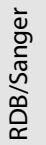

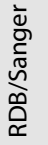

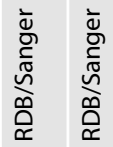

ֻ

$\stackrel{0}{x}$

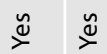

$\stackrel{y}{x}$

$\stackrel{0}{x}$

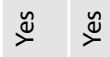

$\underset{\substack{a \\ \stackrel{m}{n}}}{\underline{n}}$

$\stackrel{\bar{d}}{\stackrel{d}{d}}$

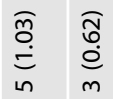

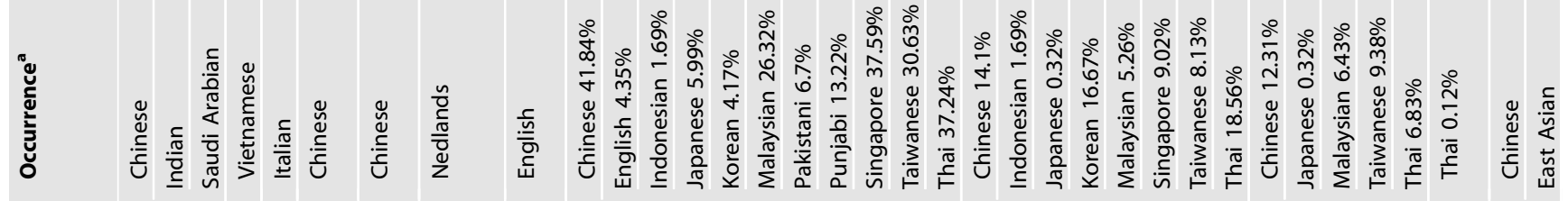

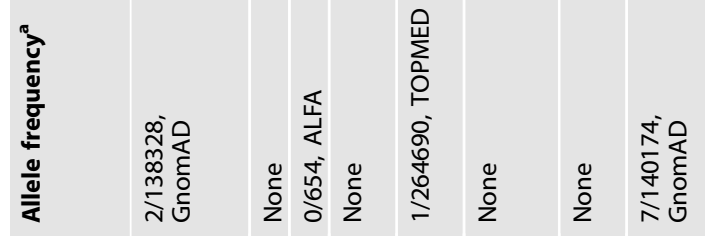

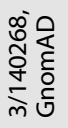

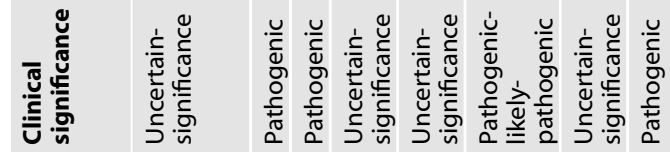

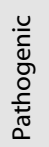

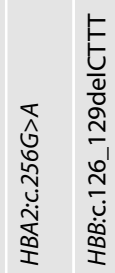

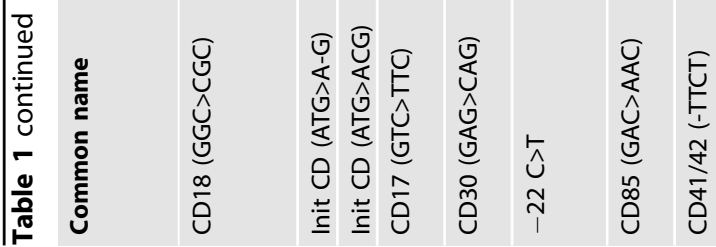
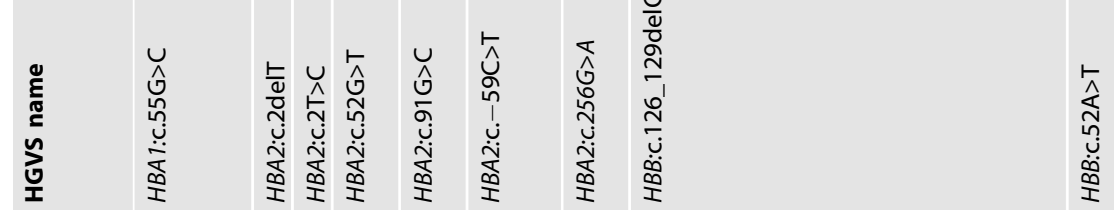

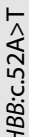

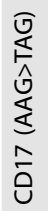

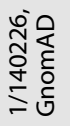

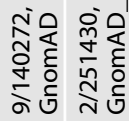

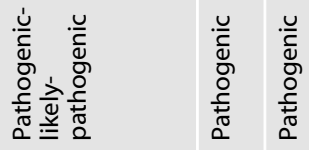

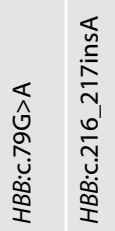

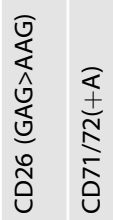




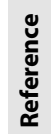

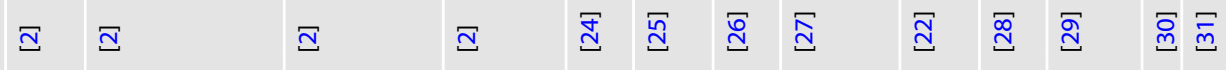

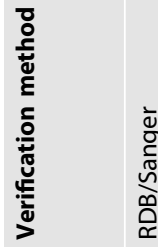

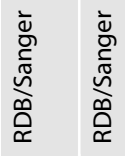

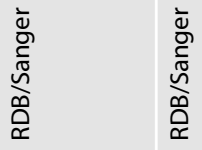

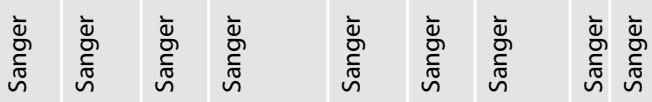

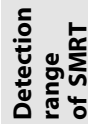

ธ。

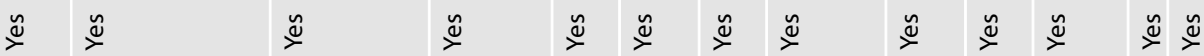

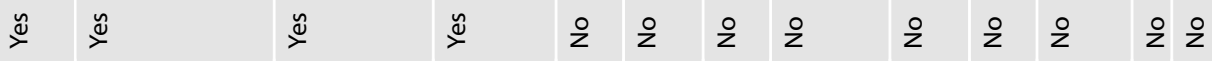

$\frac{a}{\stackrel{n}{a}}$

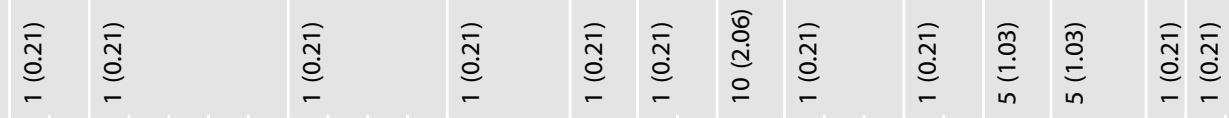

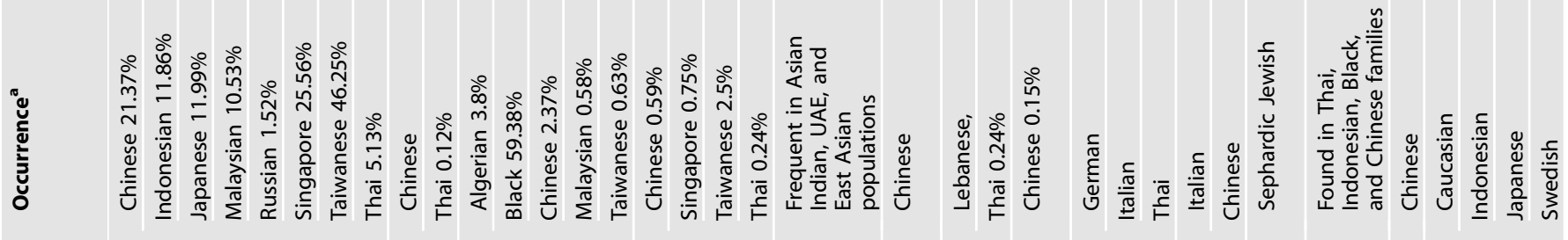

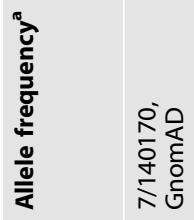

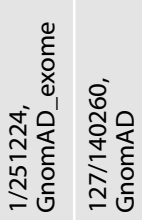

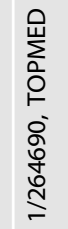

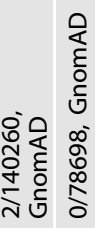

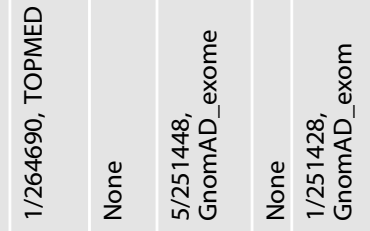

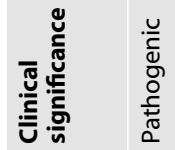

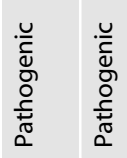

$\frac{0}{\pi}$
$\frac{\pi}{5}$
$\frac{\pi}{2}$
$\frac{\pi}{4}$

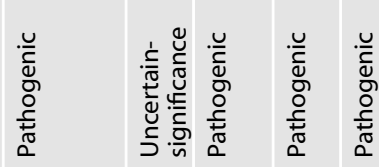

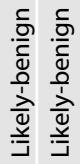

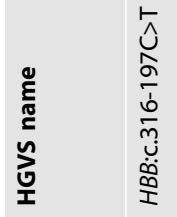

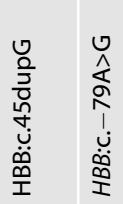

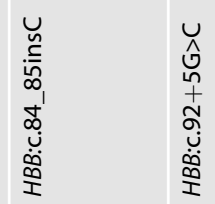

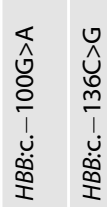

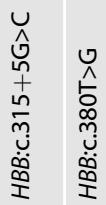

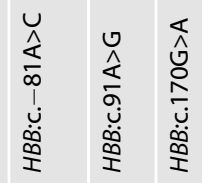

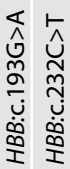

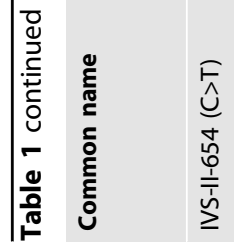

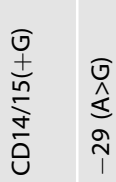

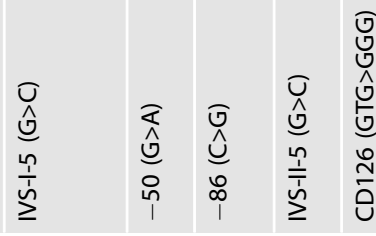

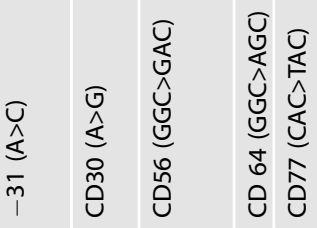




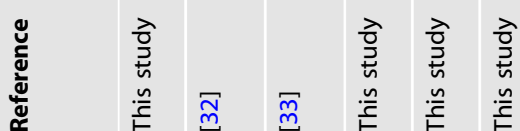

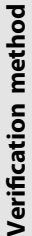

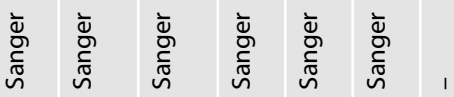
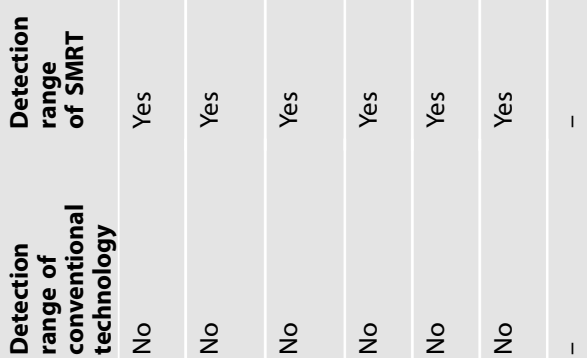

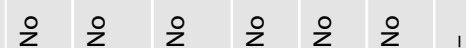

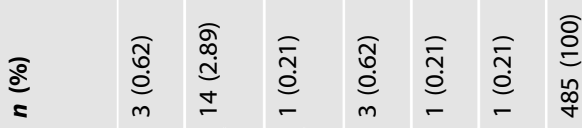
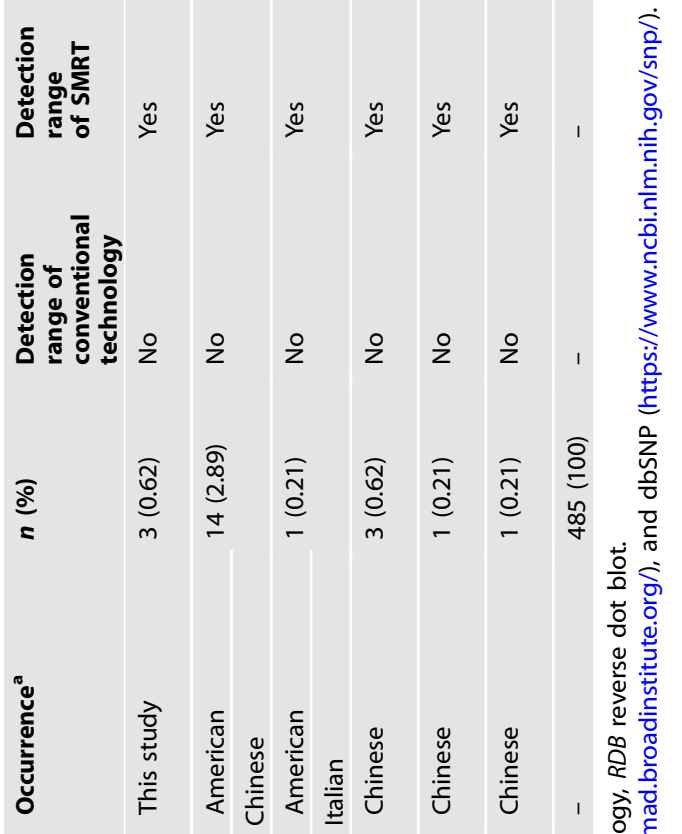

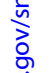

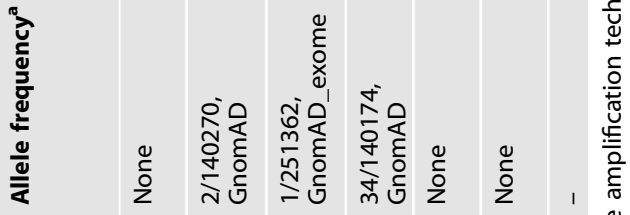

Table 2. Genotype analysis with exactly the same test results for the two types of technologies.

\begin{tabular}{|c|c|c|c|}
\hline SMRT & $\begin{array}{l}\text { Conventional } \\
\text { technologies }\end{array}$ & Concordance & $n$ \\
\hline$-\alpha^{4.2} / \alpha \alpha$ & $-\alpha^{4.2} / \alpha \alpha$ & Yes & 104 \\
\hline$-\alpha^{3.7} / \alpha \alpha$ & $-\alpha^{3.7} / \alpha \alpha$ & Yes & 43 \\
\hline$-{ }_{-} \mathrm{SEA} /-\alpha^{3.7}$ & $-{ }^{-S E A} /-\alpha^{3.7}$ & Yes & 32 \\
\hline$-{ }_{-}^{\mathrm{SEA}} / \alpha \alpha$ & $--_{\mathrm{SEA}} / \alpha \alpha$ & Yes & 24 \\
\hline$-{ }^{\mathrm{SEA}} /-\alpha^{4.2}$ & $-{ }^{\mathrm{SEA}} /-\alpha^{4.2}$ & Yes & 13 \\
\hline$\alpha^{C S} \alpha / \alpha \alpha$ & $\alpha^{\mathrm{CS}} \alpha / \alpha \alpha$ & Yes & 6 \\
\hline$-\alpha^{3.7} / \alpha^{\mathrm{CS}} \alpha$ & $-\alpha^{3.7} / \alpha^{\mathrm{CS}} \alpha$ & Yes & 4 \\
\hline$-\alpha^{3.7} /-\alpha^{4.2}$ & $-\alpha^{3.7} /-\alpha^{4.2}$ & Yes & 2 \\
\hline$-\alpha^{4.2} / \alpha^{\mathrm{CS}} \alpha$ & $-\alpha^{4.2} / \alpha^{\mathrm{CS}} \alpha$ & Yes & 2 \\
\hline$\alpha^{\mathrm{Ws}} \alpha / \alpha \alpha$ & $\alpha^{\mathrm{WS}} \alpha / \alpha \alpha$ & Yes & 2 \\
\hline$-\alpha^{4.2} /-\alpha^{4.2}$ & $-\alpha^{4.2} /-\alpha^{4.2}$ & Yes & 1 \\
\hline$-{ }_{-} \mathrm{SEA} /-\alpha^{4.2}$ & $--\mathrm{SEA}^{-}-\alpha^{4.2}$ & Yes & 1 \\
\hline$--^{\mathrm{THAI}} / \alpha^{\mathrm{CS}} \alpha$ & $--^{\mathrm{THAl}} / \alpha^{\mathrm{CS}} \alpha$ & Yes & 1 \\
\hline$\alpha^{\mathrm{CS}} \alpha / \alpha^{\mathrm{QS}} \alpha$ & $\alpha^{\mathrm{CS}} \alpha / \alpha^{\mathrm{QS}} \alpha$ & Yes & 1 \\
\hline$\alpha^{Q S} \alpha / \alpha \alpha$ & $\alpha^{\mathrm{QS}} \alpha / \alpha \alpha$ & Yes & 1 \\
\hline$\beta^{\mathrm{CD} 17(\mathrm{AAG}>\mathrm{TAG})} / \beta^{\mathrm{N}}$ & $\beta^{\mathrm{CD} 17(\mathrm{AAG}>\mathrm{TAG})} / \beta^{\mathrm{N}}$ & Yes & 9 \\
\hline$\beta^{\mathrm{CD} 41 / 42(-\mathrm{TTCT})} / \beta^{\mathrm{N}}$ & $\beta^{\mathrm{CD} 41 / 42(-T T C T)} / \beta^{N}$ & Yes & 9 \\
\hline$\beta_{\text {AAG }>\text { TAG) }}^{\mathrm{CD} 41 / 42(-\mathrm{TTCT})} / \beta^{\mathrm{CD} 17}$ & $\beta_{\mathrm{AAG}>\mathrm{TAG})}^{\mathrm{CD} 41 / 42(-\mathrm{TTCT})} / \beta^{\mathrm{CD} 17}$ & Yes & 2 \\
\hline$\beta^{\mathrm{CD} 71 / 72(+\mathrm{A})} / \beta^{\mathrm{N}}$ & $\beta^{\mathrm{CD} 71 / 72(+\mathrm{A})} / \beta^{\mathrm{N}}$ & Yes & 2 \\
\hline$\beta^{\mathrm{CD} 26(\mathrm{GAG}>A A G)} / \beta^{N}$ & $\beta^{\mathrm{CD} 26(G A G>A A G)} / \beta^{N}$ & Yes & 2 \\
\hline$\beta^{-28(A>G)} / \beta^{-28(A>G)}$ & $\beta^{-28(A>G)} / \beta^{-28(A>G)}$ & Yes & 1 \\
\hline$\beta^{\mathrm{CD} 14 / 15(+\mathrm{G})} / \beta^{\mathrm{N}}$ & $\beta^{\mathrm{CD} 14 / 15(+\mathrm{G})} / \beta^{\mathrm{N}}$ & Yes & 1 \\
\hline$\beta_{A>G)}^{C D 41 / 42(-T T C T)} / \beta^{-28}$ & $\beta^{\mathrm{CD} 41 / 42(-T T C T)} / \beta^{-28(\mathrm{~A}>\mathrm{G})}$ & Yes & 1 \\
\hline$\beta^{\text {IVS-I-5(G>C) } / \beta^{N}}$ & $\beta^{\mathrm{IVS}-1-5(\mathrm{G}>\mathrm{C})} / \beta^{\mathrm{N}}$ & Yes & 1 \\
\hline$\beta^{\mathrm{IVS}-11-654(\mathrm{C}>\mathrm{T})} / \beta^{\mathrm{N}}$ & $\beta^{\mathrm{IVS}-1 \mathrm{II}-654(\mathrm{C}>\mathrm{T})} / \beta^{\mathrm{N}}$ & Yes & 1 \\
\hline$\beta_{A>G)}^{I V S-1 I-654(C>T)} / \beta^{-28}$ & $\beta^{\text {IVS-II-654(C>T) }} / \beta^{-28(A>G)}$ & Yes & 1 \\
\hline$\beta^{-29(A>G)} / \beta^{N}$ & $\beta^{-29(A>G)} / \beta^{N}$ & Yes & 1 \\
\hline$\beta^{-28(A>G)} / \beta^{N}$ & $\beta^{-28(A>G)} / \beta^{N}$ & Yes & 1 \\
\hline $\begin{array}{l}\alpha_{(A A G>T A G)}^{C S} \alpha / \beta^{-28(A>G)} \text { with } \beta^{C D 17} \\
{ }_{(A A G)}^{C D} \alpha\end{array}$ & $\begin{array}{l}\alpha^{C S} \alpha / \alpha \alpha \text { with } \beta^{C D} \beta^{C D} 17 \\
(A A G T A G) / \beta^{-28(A>G)}\end{array}$ & Yes & 1 \\
\hline $\begin{array}{l}-\mathrm{zEA}^{\mathrm{SEA}} / \alpha \alpha \text { with } \beta^{\mathrm{CD} 41 /} \\
42(-\mathrm{TTCT}) / \beta^{-28(\mathrm{~A}>\mathrm{G})}\end{array}$ & $\begin{array}{l}- \text {-SEA }_{(-\pi \alpha} \alpha \text { with } \beta^{\mathrm{CD} 41 / 42} \\
(-\mathrm{TTCT}) / \beta^{-28(\mathrm{~A}>\mathrm{G})}\end{array}$ & Yes & 2 \\
\hline $\begin{array}{l}\alpha_{(A)}^{Q Q S} \alpha \alpha \alpha \text { with } \beta^{C D 17} \\
(A A G) / \beta^{N}\end{array}$ & $\begin{array}{l}\alpha^{\mathrm{QS}} \alpha / \alpha \alpha \text { with } \beta^{\mathrm{CD} 17} \\
(\mathrm{AAG}>\mathrm{TAG}) / \beta^{\mathrm{N}}\end{array}$ & Yes & 1 \\
\hline $\begin{array}{l}\text { SEA } / \alpha \alpha \text { with } \\
\text { (A>G) } / \beta^{-28(A>G)} \beta^{-28}\end{array}$ & $\begin{array}{l}- \text {-SEA }_{/} \alpha \alpha \text { with } \beta^{-28(\mathrm{~A}>\mathrm{G})} / \\
\beta^{-28(\mathrm{~A}>\mathrm{G})}\end{array}$ & Yes & 1 \\
\hline $\begin{array}{l}-{ }_{-5 E A}^{\mathrm{SEA}} / \alpha \alpha \text { with } \beta^{\mathrm{CD} 41 /} \\
42(-\mathrm{TTCT}) / \beta^{\mathrm{CD} 41 / 42} \\
(- \text { TTCT) }\end{array}$ & 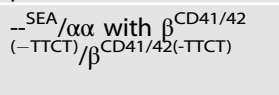 & Yes & 1 \\
\hline 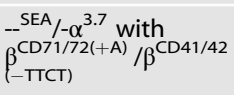 & $\begin{array}{l}-\mathrm{SEA} /-\alpha^{3.7} \text { with } \beta^{\mathrm{CD} 71 / 72} \\
(+\mathrm{A}) / \beta^{\mathrm{CD} 41 / 42(-\Pi \mathrm{CT})}\end{array}$ & Yes & 1 \\
\hline $\begin{array}{l}-{ }^{\mathrm{SEA}} / \alpha^{3.7} \text { with } \beta^{\mathrm{CD} 17} \\
(\mathrm{AAG}>\mathrm{TAG}) / \beta^{\mathrm{N}}\end{array}$ & $\begin{array}{l}-{ }^{\mathrm{SEA}} /-\alpha^{3.7} \text { with } \beta^{\mathrm{CD} 17} \\
(\mathrm{AAG}>\mathrm{TAG}) / \beta^{\mathrm{N}}\end{array}$ & Yes & 1 \\
\hline 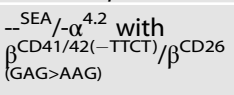 & $\begin{array}{l}-\mathrm{SEA} / \alpha^{4.2} \text { with } \beta^{\mathrm{CD} 41 / 42} \\
(-\mathrm{TTCT}) / \beta^{\mathrm{CD} 26(\mathrm{GAG}>\mathrm{AAG})}\end{array}$ & Yes & 1 \\
\hline $\begin{array}{l}-\alpha^{3.7} / \alpha^{\mathrm{CS}} \alpha \text { with } \\
\beta^{\mathrm{CD} 26(\mathrm{GAG}>A A G)} / \beta^{\mathrm{N}}\end{array}$ & $\begin{array}{l}-\alpha^{3.7} / \alpha^{\mathrm{CS}} \alpha \text { with } \beta^{\mathrm{CD} 26} \\
\text { (GAG>AAG)} / \beta^{N}\end{array}$ & Yes & 1 \\
\hline $\begin{array}{l}-\alpha^{4.2} / \alpha \alpha \text { with } \beta^{\mathrm{CD} 41 /} \\
42(-\mathrm{TTCT}) / \beta^{\mathrm{CD} 1} \\
\text { (AAG>TAG) }\end{array}$ & $\begin{array}{l}-\alpha^{4.2} / \alpha \alpha \text { with } \beta^{\mathrm{CD} 41 / 42} \\
(-\mathrm{TTCT}) / \beta^{\mathrm{CD} 17(\mathrm{~A} A \mathrm{AG}>\mathrm{TAG})}\end{array}$ & Yes & 1 \\
\hline $\begin{array}{l}-\alpha^{4.2} / \alpha \alpha \text { with } \beta^{\mathrm{CD} 26} \\
(\mathrm{GAG}>\mathrm{AAG}) / \beta^{\mathrm{N}}\end{array}$ & $\begin{array}{l}-\alpha^{4.2} / \alpha \alpha \text { with } \beta^{\mathrm{CD} 26} \\
(\mathrm{GAG}>\mathrm{AAG}) / \beta^{\mathrm{N}}\end{array}$ & Yes & 1 \\
\hline Total & - & - & 281 \\
\hline
\end{tabular}



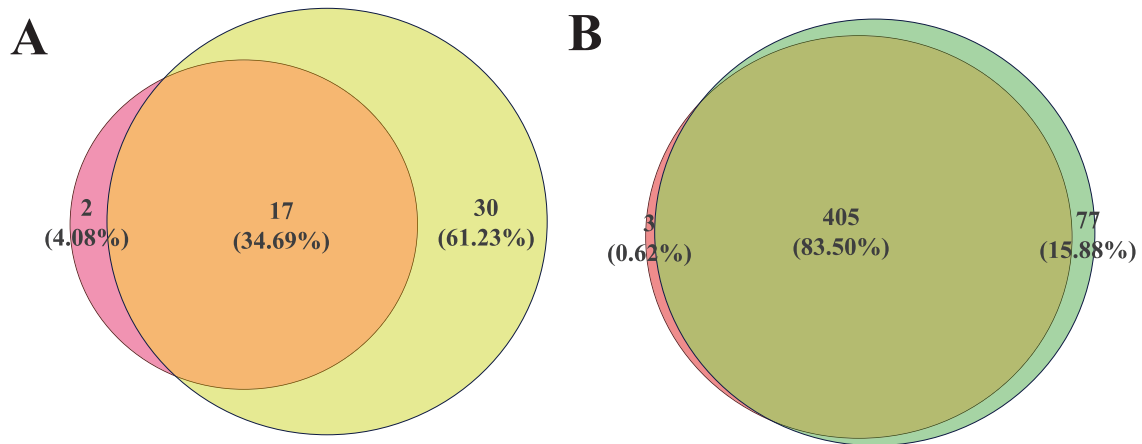

Fig. 3 Distribution of $\mathbf{4 9}$ variant types and $\mathbf{4 8 5}$ positive alleles. A Distribution of 49 variant types. SMRT cannot detect but conventional techniques can detect (pink), SMRT and conventional techniques can detect (orange), SMRT can detect but conventional techniques failto detect (yellow). B Distribution of 485 positive alleles. SMRT cannot detect but conventional techniquescan detect (red), SMRT and conventional techniques can detect (yellow-green), SMRT can detect butconventional techniques fail to detect (light green).

\section{Data analysis}

The subjects with positive test results were diagnosed as carriers or patients of thalassemia. The concordance of the two methods were then calculated. SPSS statistical software for Windows, version 22.0 (SPSS Chicago, IL, USA) was used for statistical analyses. The measurement data are expressed as $\mathrm{X} \pm \mathrm{SD}$ and the count data are expressed as examples and percentages.

\section{RESULTS}

\section{Comparison of genotyping results between traditional} methods and SMRT technology

Among the 434 enrolled cases, using conventional methods (gapPCR and /or MLPA, RDB), 318 patients with variants (73.27\%), and 116 patients without variants $(26.73 \%)$ were identified. SMRT identified 361 patients with variants (83.18\%) and 73 patients without variants $(16.82 \%)$. The positive detection rate of SMRT was $9.91 \%$ higher than that of conventional methods. Combined analyses of the two techniques revealed that there were 485 positive alleles among 49 variant types (Fig. 1). Among them, conventional technology identified 408 positive alleles (84.12\%) among 19 variant types (38.78\%), and SMRT identified 482 positive alleles (99.38\%) among 47 variant types (95.92\%) (Fig. 2). Of the 49 types of variants, 30 types $(61.23 \%)$ were detected by SMRT only, two types (4.08\%) were detected by conventional methods only, and 17 types (34.69\%) were detected by both methods (Fig. 2). Of the 485 positive alleles, 77 (15.88\%) were identified by SMRT only, three $(0.62 \%)$ were identified by conventional methods and 405 $(83.50 \%)$ were identified by both methods (Table 1 and Fig. 2). A total of 354 cases had completely concordant results between the two types of techniques, including 73 negative and 281 positive cases (Table 2 and Fig. 3), while 80 cases had discordant results (Fig. 3). Of the 80 cases, 14 patients had rare deletions and triplicate a-globin genes (Table 3), 16 patients had rare variants in the a-globin gene (Table 4), and 49 patients had rare variants in the $\beta$-globin gene (Table 5), one case had $a^{3.7}$ deletion. The genotype of sample D141966 was $-a^{3.7} / a a$ by SMRT method, while it was $-a^{3.7} /-a^{3.7}$ by conventional Gap-PCR. Validated by MLPA technology, the genotype is the same as the result of SMRT technology (Fig. 4). The IGV plots of selected samples were displayed to show the thalassemia variants identified by SMRT (Fig. 5).

\section{Hematology examination and hemoglobin electrophoresis results in patients with rare deletions and triplicate a-globin genes}

A total of 14 rare cases of a and $\beta$-globin gene deletions, or triplicate a-globin genes were found (Table 3). Among them, the result of hemoglobin electrophoresis of patients with $-\mathrm{SEA}^{\mathrm{S}} /-\mathrm{a}^{2.4}$ and $-a^{3.7} / \mathrm{HS}-40$ deletion showed the presence of $\mathrm{HBH}$ peak.
The routine hematology examination results of patients with aa/ $\mathrm{aaa}^{\text {anti3.7 }}$ and aa/aaa ${ }^{\text {anti4.2 }}$ were normal, but $\mathrm{HbA} 2$ was decreased and $\mathrm{HbF}$ was significantly increased to $12.3 \%$ and $16.2 \%$, respectively. When aa/aaa ${ }^{\text {anti3.7 }}$ and $\mathrm{aa} / \mathrm{aaa}^{\text {anti4.2 }}$ were combined with $\beta$-thalassemia, they were assigned as intermediate $\beta$-thalassemia, and $\mathrm{HbF}$ was also significantly increased. Patients with $\mathrm{HKaa} / \mathrm{aa}$ presented with silent a-thalassemia, and patients with $\mathrm{HKaa} /-\mathrm{SEA}$ presented with mild a-thalassemia. The HBG1$H B G 2$ deletion combined with $\beta^{\mathrm{CD} 41 / 42(-\mathrm{TTCT})} / \beta^{N}$ was clinically manifested as mild $\beta$-thalassemia (Table 3 ).

\section{Hematology examination and hemoglobin electrophoresis} results in patients with rare variants in $H B A 1 / 2$

Sixteen rare $H B A 1 / 2$ variants were found, including $-\mathrm{SEA}_{\mathrm{C}} / \mathrm{HBA2}$ : c.2delT, $\quad-{ }_{-}$SEA $/ H B A 2: C .2 T>C,--{ }^{-}$SEA $/ H B A 2: C .52 \mathrm{G}>\mathrm{T},-\mathrm{a}^{3.7} / \mathrm{HBA1}: \mathrm{c} .19 \mathrm{G}>\mathrm{T}$, HBA 1:C.34A >C,HBA1:C.51G>C,HBA1:C.55G>C,--- SEA /HBA1:C.55G>C,--SEA/ HBA1:c.84G>T,HBA2:C.91G>C,HBA2:C. $-59 \mathrm{C}>\mathrm{T}$ combined with HBA2: c.91G>C, HBA2:c.256G>A. Among them, HBA1: c.34A>C and HBA1: c.51G $>C$ had normal routine blood test results, but abnormal hemoglobin was detected. The routine blood phenotypes of the genotypes HBA1: c.84G>T, HBA1:C.19G>T, and HBA1:C.55G>C were normal. If it was compounded with other deletion variant, it manifested as mild or silent thalassemia, and abnormal hemoglobin

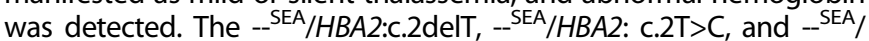
HBA2: c.52G $>\mathrm{T}$ manifested as non-deletion $\mathrm{HbH}$ disease. $H B A 2$ : c.91G $>C$ had normal hematology phenotype, though with abnormal hemoglobin, and reduced $\mathrm{HbA2}$. In cases with HBA2: c. $-59 \mathrm{C}>\mathrm{T} /$ HBA2: c.91G>C compound heterozygous variants, hemoglobin electrophoresis showed abnormal hemoglobin peaks at 3.784 and $4.349 \mathrm{~min}$, the routine hematology phenotype was normal, and $\mathrm{HbA} 2$ was significantly increased. HBA2: c.256G $>$ A cases had a normal phenotype, though abnormal hemoglobin was detected, and $\mathrm{HbA} 2$ was reduced (Table 4).

\section{Hematology examination and hemoglobin electrophoresis results in patients with rare variants in $H B B$}

The c. $-100 \mathrm{G}>\mathrm{A}$, c. $-136 \mathrm{C}>\mathrm{G}, \mathrm{c} .315+5 \mathrm{G}>\mathrm{C}, \mathrm{c} .380 \mathrm{~T}>\mathrm{G}$, and c. $-81 \mathrm{~A}>\mathrm{C}$ belonged to beta + thalassemia (partial loss of function of $\beta$-globin gene). Heterozygous variants of these types showed silent or mild $\beta$-thalassemia. $c .91 \mathrm{~A}>\mathrm{G}$ belonged to beta0 thalassemia (complete loss of function of the $\beta$-globin gene), and heterozygous mutations manifested as mild $\beta$ thalassemia. The hematological phenotype of $c .170 \mathrm{G}>\mathrm{A}, \mathrm{c} .431 \mathrm{~A}>\mathrm{G}, \mathrm{c} .232 \mathrm{C}>\mathrm{T}$, c. $341 \mathrm{~T}>\mathrm{A}$, and c.431A $>\mathrm{G}$ heterozygous variation was normal, the $\mathrm{HbA} 2$ and $\mathrm{HbF}$ contents were within the reference range, but abnormal hemoglobins were detected. Abnormal hemoglobin of case with c.341T $>A / c .315+5 G>C$ accounted for $93.5 \%$, and $\mathrm{HbA}$ was almost undetectable. $\mathrm{HbA} 2$ content of case with $\mathrm{c.431A}>\mathrm{G}$ heterozygous variation was increased. The hematological 
‡

星 $\bar{i}, \stackrel{m}{\text { ก }}$

ஓ

㭊 ப.

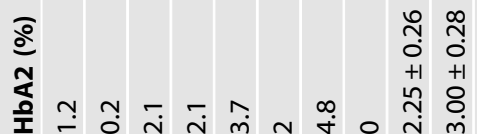

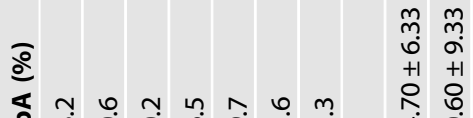

产

$\dot{\mathfrak{g}}$

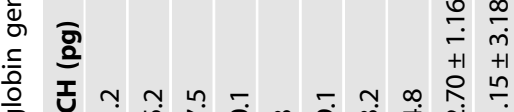

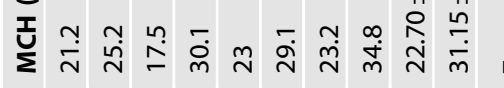

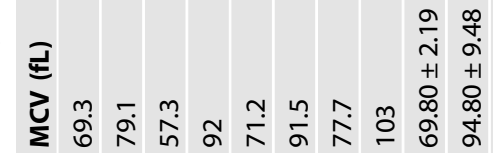

క⿱一兀)

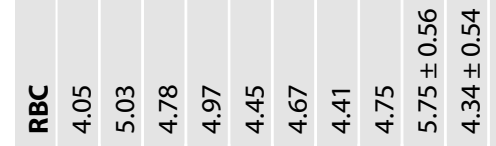

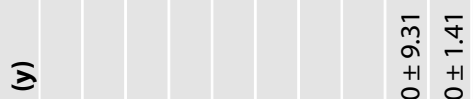

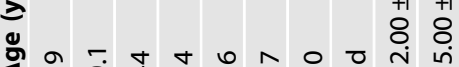

ष্े

$=-t--t-t h \pi$

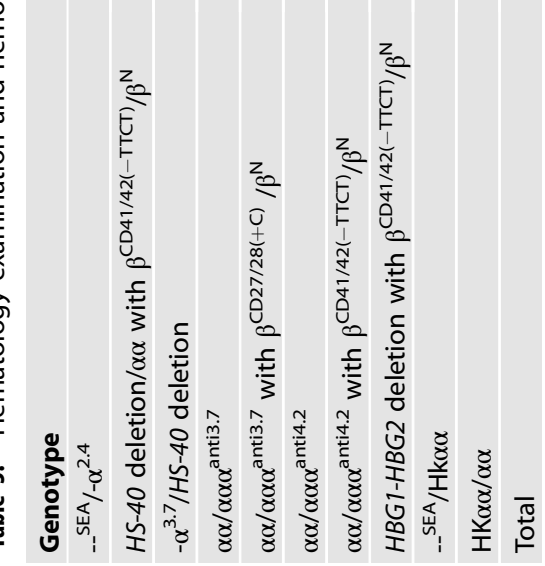

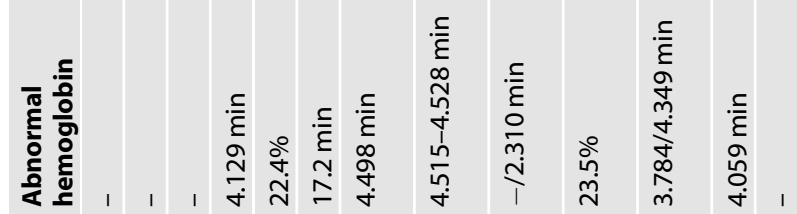

๖

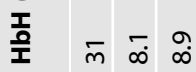

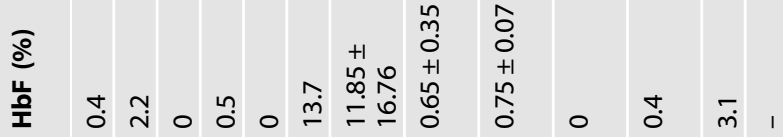

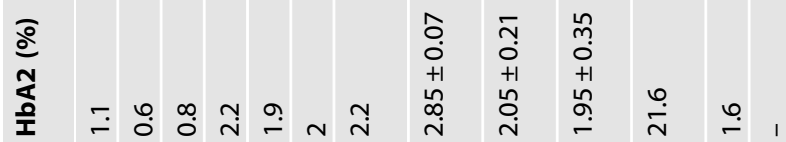

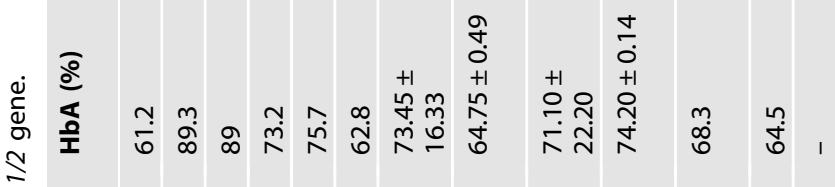

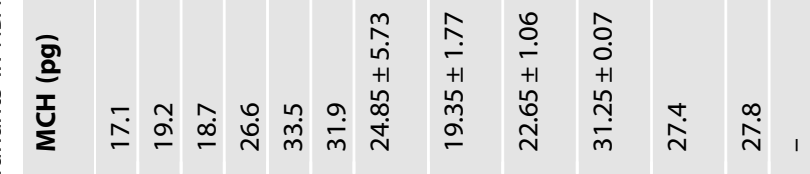

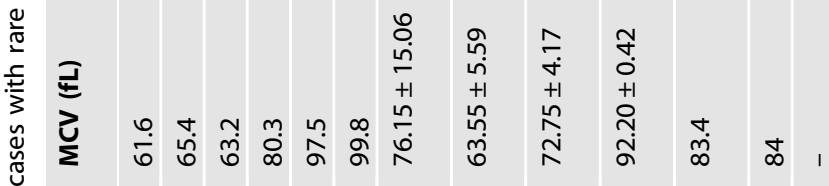

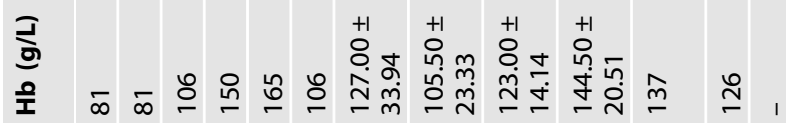

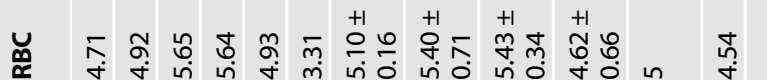
$\frac{0}{0}$

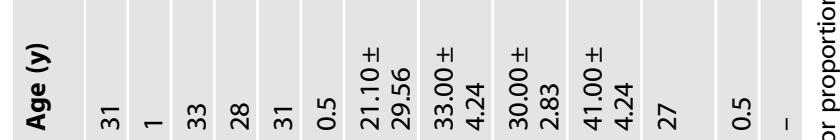

$\stackrel{\frac{0}{2}}{\stackrel{0}{\frac{0}{0}}}$

ปั

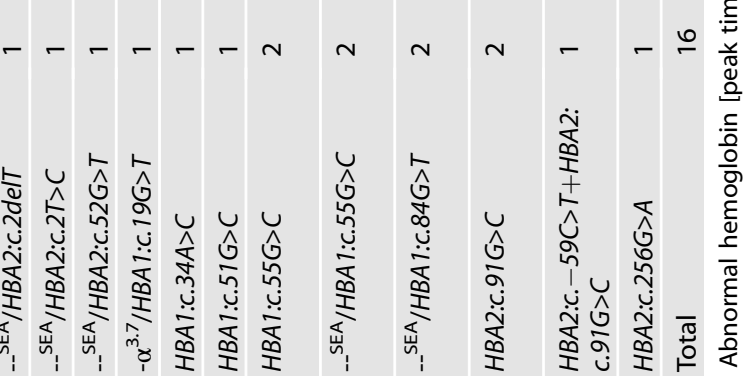




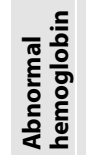

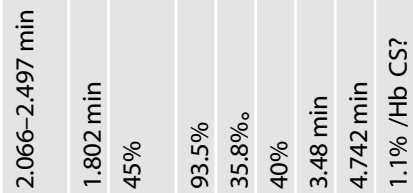

$\frac{\grave{9}}{\frac{0}{9}}$

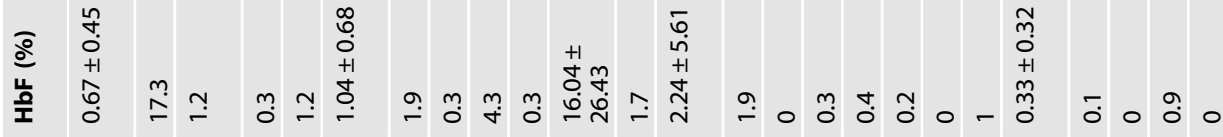

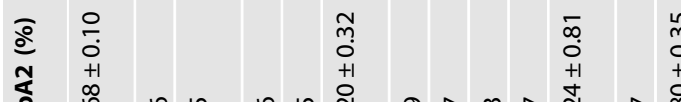

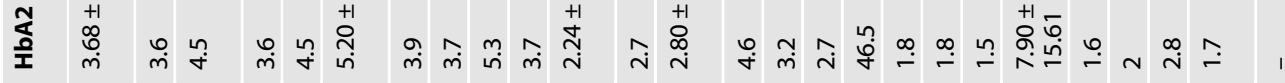

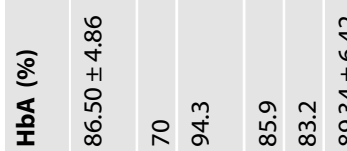

$\stackrel{\infty}{\infty}$

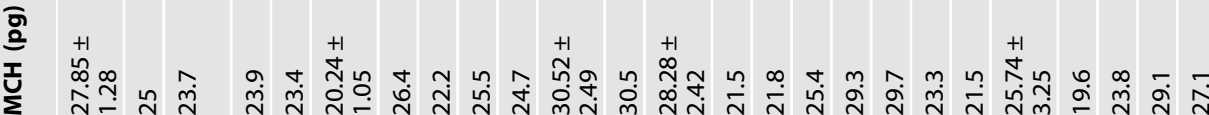

茓

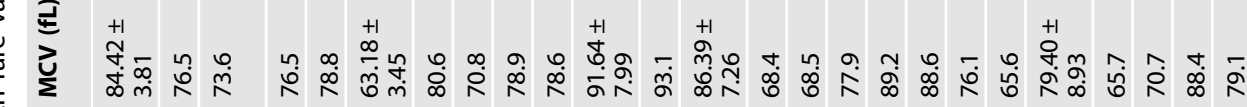

妾

离

$\underset{\substack{n \\ \stackrel{m}{*}}}{\stackrel{+}{4}}$

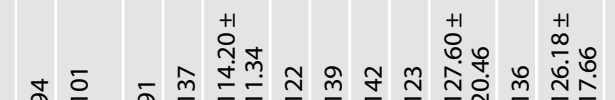

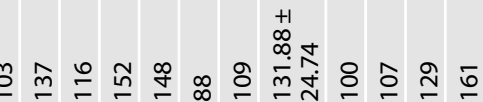

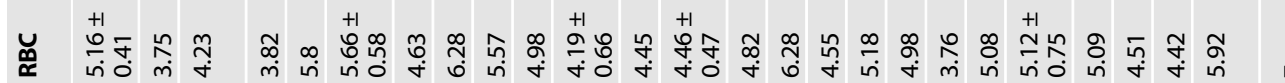

产

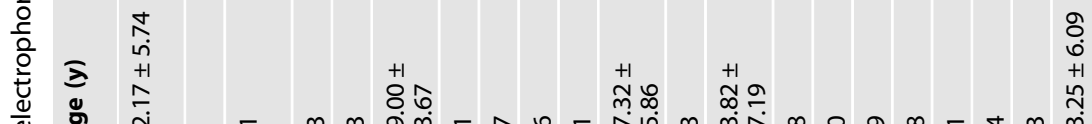

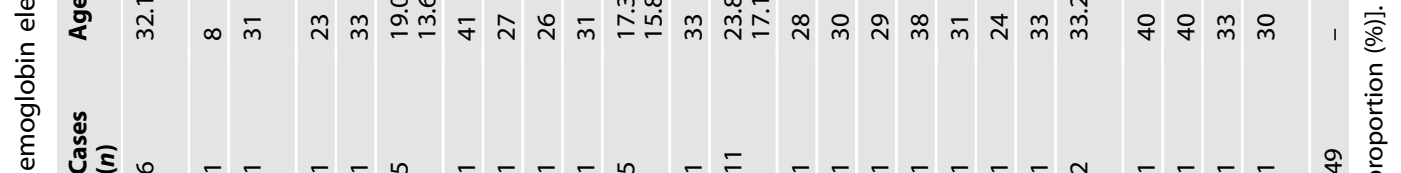

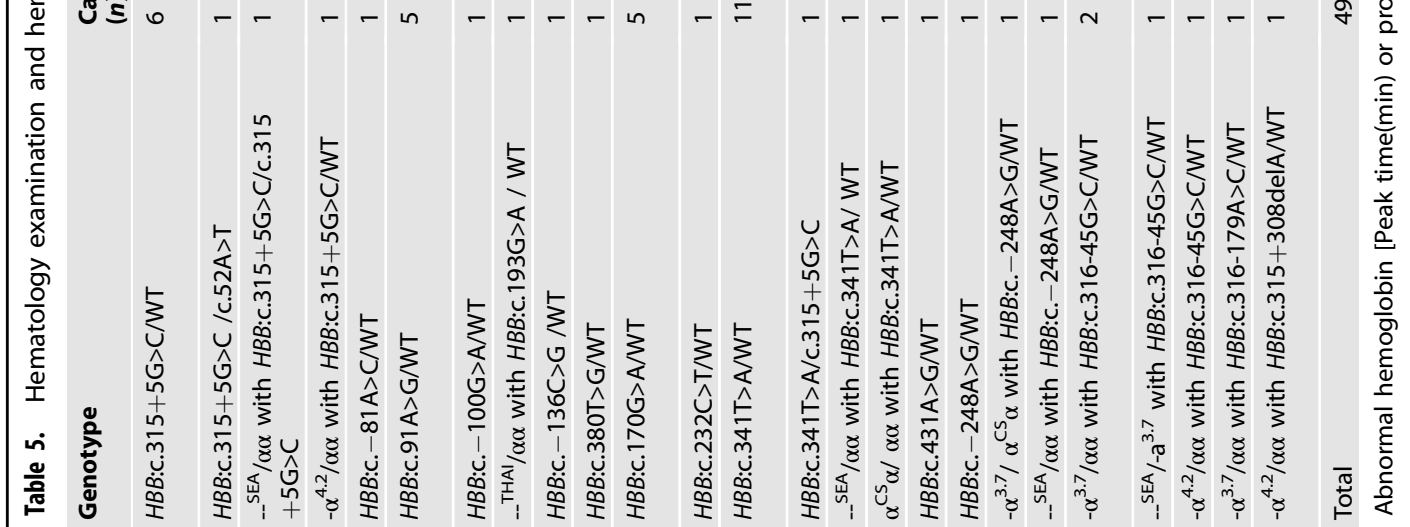



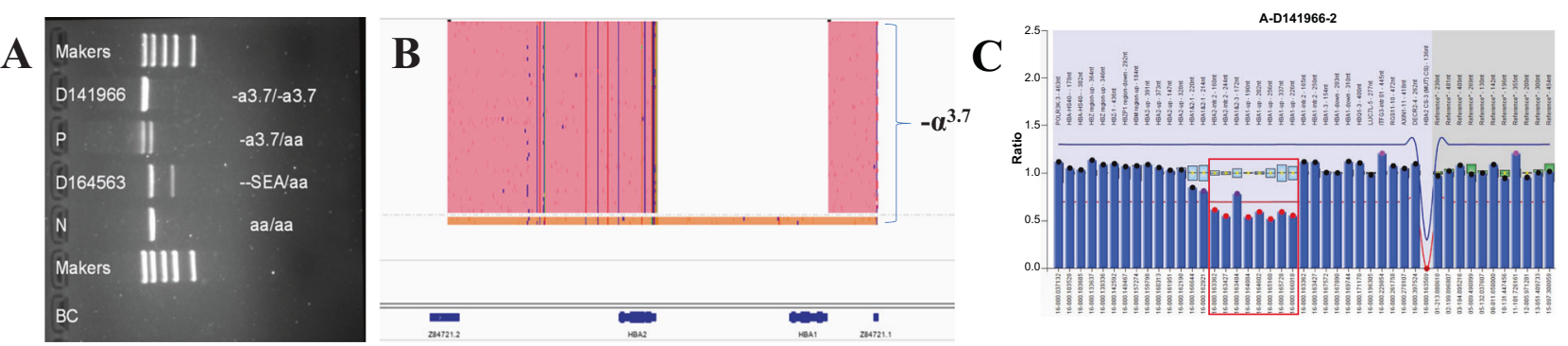

Fig. 4 The genotyping results of sample D141966 by three technologies. A It was $-\alpha^{3.7} /-\alpha^{3.7}$ by Gap-PCR. B It was $-\alpha^{3.7} / \alpha \alpha$ by SMRT. C It was $-\alpha^{3.7} / \alpha \alpha$ by MLPA. Redboxed areas indicate the position of $-\alpha^{3.7}$ deletion.

phenotype of c. $-248 \mathrm{~A}>\mathrm{G}$ heterozygous variation was normal, and mainly manifested as decreased $\mathrm{HbA} 2$ content. c.316-45G $>\mathrm{C}$, c.316-179A >C, and c.315+308delA combined with other types of a-thalassemia were manifested as silent or mild a-thalassemia (Table 5).

\section{DISCUSSION}

Thalassemia is a single gene disease, which is difficult to cure but more straightforward to diagnose and be prevented clinically. Its gene mutation types are diverse and complex. As of 2021, the LOVD (https://databases.lovd.nl/shared/genes) database had more than 2000 thalassemia and abnormal hemoglobin-related variant sites, and most of the sites have not been studied by conventional genetic testing methods, especially the large deletion variant type. At present, common clinical testing techniques for thalassemia genes include Gap-PCR, reverse dot hybridization, PCR-flow fluorescence hybridization, gene chip, MLPA, Sanger sequencing, and next generation sequencing. The conventional screening mode can only detect variants in known gene loci, which is far from sufficient for the detection of other variant loci, leading to missed diagnoses and misdiagnoses. There is therefore an urgent need to use more accurate and effective diagnostic techniques to screen thalassemia patients in clinical practice. In recent years, there have been reports of missed detections of thalassemia using conventional genetic testing methods. The SMRT technology can detect the thalassemia gene without interrupting the DNA, and can directly read the full-length gene sequence. The DNA does not need to be amplified by PCR during sequencing, which facilitates individual sequencing of each DNA molecule, and it has very long read lengths (a read length up to $30-100 \mathrm{~kb}$ ), high accuracy (QV30 > 99.8\%), no GC preference, and single-molecule resolution characteristics [7]. SMRT technology can facilitate the simultaneous detection of $\alpha$-thalassemia and $\beta$-thalassemia in $1 \mu \mathrm{L}$ of whole blood or $10-15 \mathrm{~mL}$ of amniotic fluid sample. It can also detect hotspots and rare variant sites and their arrangements with high accuracy, including comprehensive coverage of 2062 variant sites related to thalassemia, and detection of 18 a-globin gene deletion variants, four a-globin genes triplicate and two $\beta$-globin gene deletion. It can detect 96 samples at a time with high efficiency and high accuracy.

$\mathrm{Xu}$ et al. [5] first used the SMRT to sequence full-length thalassemia-related genes (HBA1/2 and $H B B)$ to obtain complete variant information of two alleles that were difficult to obtain by conventional genetic testing techniques. Twelve hospitals in southern China assessed a comprehensive analysis of thalassemia alleles (CATSA) for identifying both $a$ and $\beta$ thalassemia genetic carrier status by third-generation sequencing (TGS). Compared with standard thalassemia variant PCR panel testing, TCS can detected 33 more positive variants, and found that the traditional PCR detection technology had 1 false negative and 8 false positive result [6]. The present study used the SMRT and conventional technologies to test the thalassemia gene in the thalassemia screening positive population in this area. The results showed that the percentage of thalassemia gene was high and the genotype was complex, rare variant types of thalassemia and the phenotypes were diverse. Among the 434 cases, 49 variant types were detected, of which 19 were detected by conventional technology and 47 were detected by SMRT technology. Compared with conventional technology, SMRT technology detected 28 more variant types. The positive detection of SMRT was $9.91 \%$ higher than that of conventional technology, and SMRT technology increased the detection of thalassemia genes. At present, the detection range of the reagents we used only included 2062 variant sites related to thalassemia on the $H B A 1 / 2$ and $H B B$ genes. HS-40 deletion occurs upstream of the a-globin gene cluster, and $H B G 1-H B G 2$ deletion occurs upstream of the $H B B$ gene cluster. The SMRT method developed in this study focused on detection of variants in $H B A 1, H B A 2$, and $H B B$ genes, which consisted the vast majority of thalassemia variants. With expanded primer pairs, the SMRT technology can definitely detect HS-40 and HBG1-HBG2 deletions. However, the sequencing cost will increase with more primer pairs [7]. So, it was the limit of the design of SMRT method in this study but not SMRT technology itself.

This study found 14 cases of rare deletions or triplicate a-globin genes. Among them, the $-\mathrm{SEA}_{\mathrm{SEA}} / \mathrm{a}^{2.4}$ and $-\mathrm{a}^{3.7} / \mathrm{HS}-40$ deletion patients all manifested with $\mathrm{HbH}$ disease [8, 9]. Carriers of $\mathrm{aaa}^{\text {anti3.7 }}$ and $\mathrm{aaa}^{\text {anti- } 4.2}$ had normal phenotypes, but $\mathrm{HbF}$ was significantly increased by $12.3 \%$ and $16.2 \%$, respectively, and $\mathrm{HbA} 2$ was reduced. When compounded with $\beta$-thalassemia, it can manifest as intermediate $\beta$-thalassemia due to the aggravation of the imbalance between the $a$ and $\beta$ chains, and $\mathrm{HbF}$ is also significantly increased $[10,11]$. In the present study, among the thalassemia carriers whose detection results were $-a^{3.7} / a a$ by conventional methods, two of them were found to be HKaa/aa using SMRT technology, and the misdiagnosis rate was as high as $4.17 \%(2 / 48)$. HKaa/aa patients presented with silent a-thalassemia, and HKaa/--SEA patients presented with mild a-thalassemia, which is consistent with past reports [12]. Although the HBG1HBG2 deletion combined with c.126_129delCTT/WT had two allelic variants in the $H B B$ gene, $H B G 1-H B G 2$ was functionally closed in adulthood and did not affect the expression of $\beta$ globin, so it was clinically mild $\beta$-thalassemia. SMRT method showed that the genotype of sample D141966 was $-a^{3.7} / a a$, while by conventional Gap-PCR it was $-a^{3.7} /-a^{3.7}$. Validation by MLPA confirmed D141966 had heterozygous $-a^{3.7}$ deletion. To investigate the basis of this discordance, we analyzed the SNV/indels in the aa allele identified by SMRT method and found there were three SNPs in the $3^{\prime}$-terminal of HBA2, which caused dropout of the aa allele in conventional Gap-PCR method that designed primer in this region.

This study found 16 rare $H B A$ gene variants. Among them, c.34A>C, c.51G>C, c.84G $>T$, and $c .19 \mathrm{G}>T$ were located in the $H B A 1$ gene. c.34A $>C$ and $c .51 G>C$ showed normal hematology, and 


\section{A. $\mathrm{SV}$ in $H B A 1 / 2$}
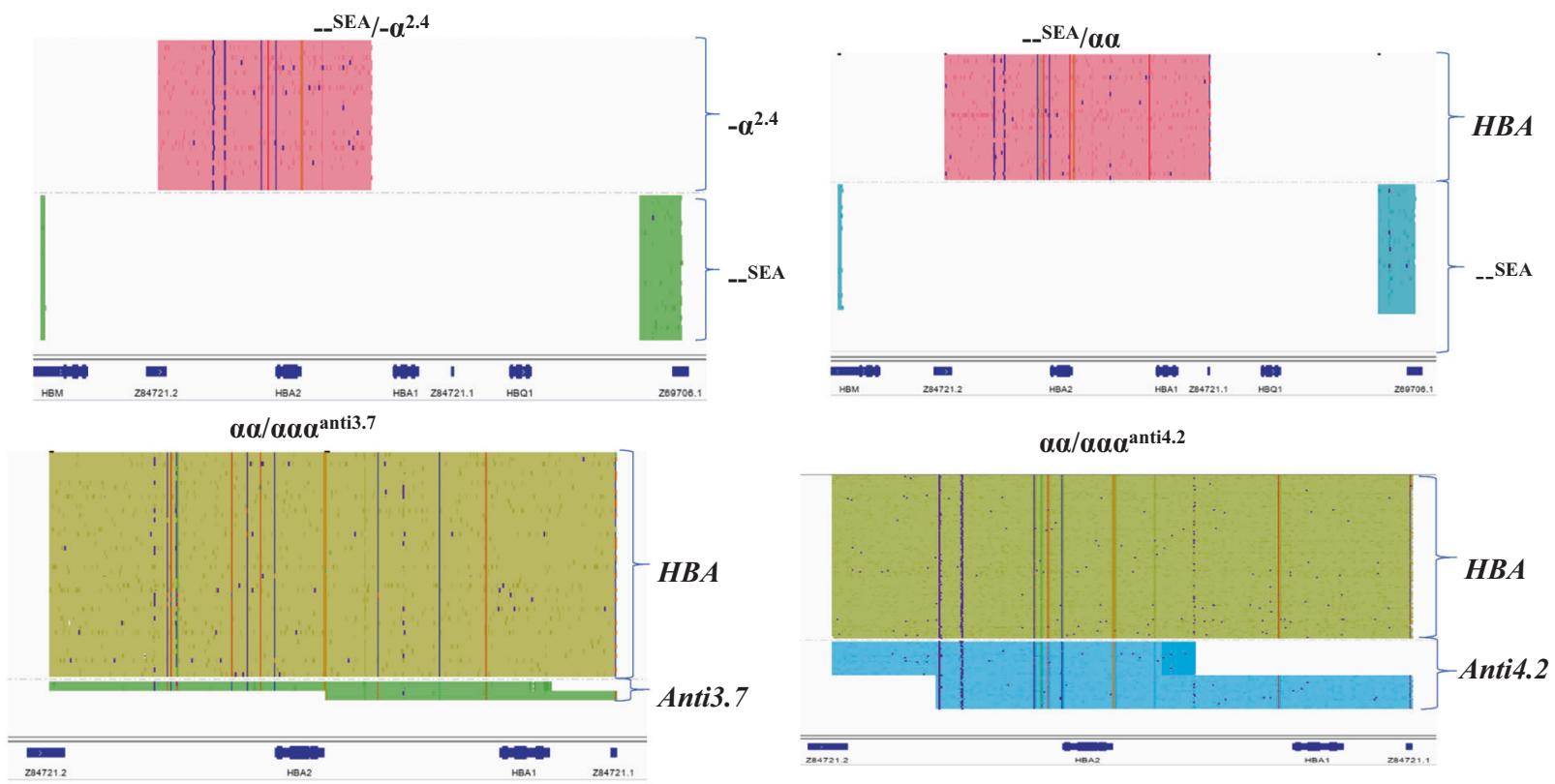

B. SNVs in $H B A 1$ and $H B B$

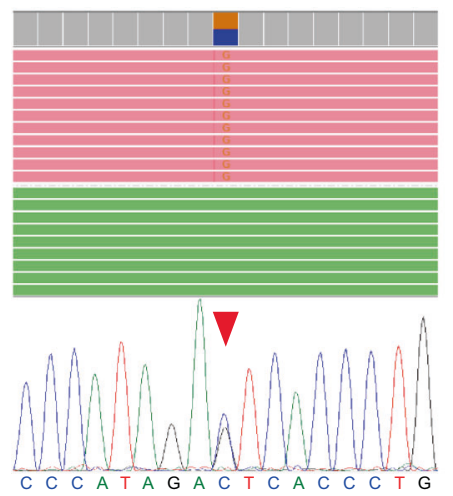

HBB:C. $315+5 G>C$
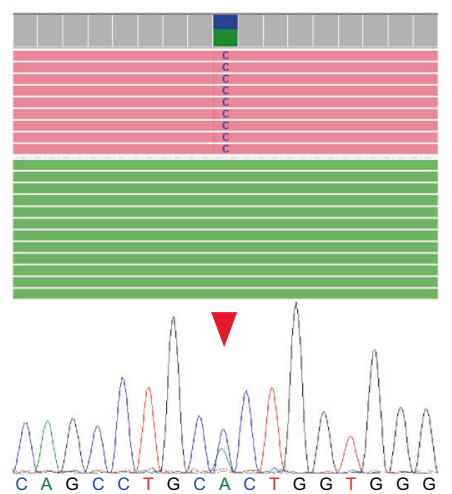

HBB:c.380T>G
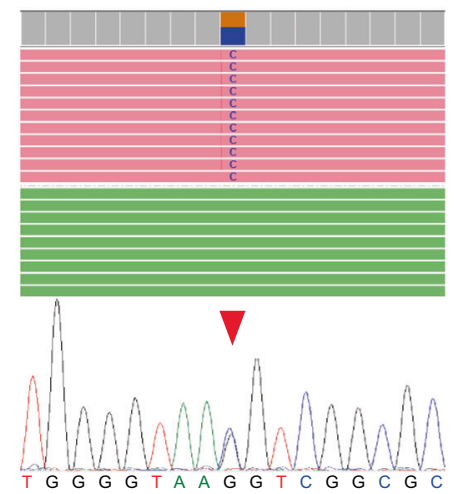

HBA1:C.51G $>\mathrm{C}$
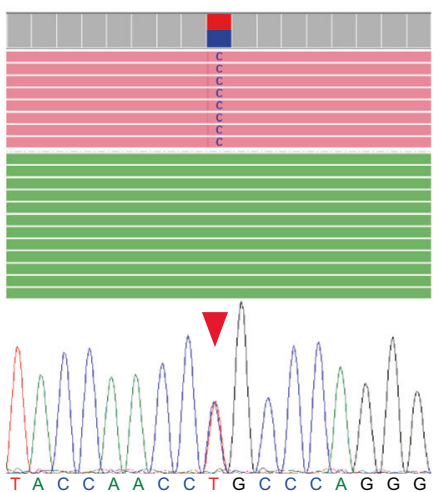

HBB:c.91A>G

Fig. 5 The Integrative Genomics Viewer plots of selected samples. Integrative Genomics Viewer plots of rare HBA1/2 structural variations (SVs; A) and single-nucleotide variations (SNVs; B) identified by comprehensive analysis of thalassemia alleles. Both the normal and variant alleles are visible in each profile. Red boxed areas indicate the position of the thalassemia variants. 
abnormal hemoglobin was detected $[13,14]$. Carriers with $H B A 1$ : C.84G>T, HBA1:C.19G>T, and HBA1:C.55G>C genotypes had a normal blood phenotype. When they were compounded with other deletion types, they could be mild or silent [15-17]. Carriers of these gene variants all showed abnormal hemoglobin, and no $\mathrm{HbH}$ phenotype was found in the compound Southeast Asian deletion.

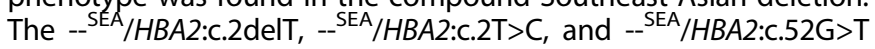
are located in the more functional $H B A 2$ gene, causing a chain synthesis to be affected, showing that the non-deletion $\mathrm{HbH}$ disease was more serious than the deletion of $\mathrm{HbH}$ disease [18-20]. HBA2: c.91G>C has a normal phenotype, the main manifestation is abnormal hemoglobin, and $\mathrm{HbA} 2$ is reduced [21]. Qadah et al. [22] reported that the $H B A 2:$ C. $-59 \mathrm{C}>\mathrm{T}$ variant caused a significant reduction in the transcription level of $H B A 2$ by $53.7 \%$. Our study reported, for the first time, $H B A 2: C .-59 C>T$ and $H B A 2$ : c.91G $>C$ compound heterozygous cases. Hemoglobin electrophoresis detected abnormal hemoglobin peaks at $3.784 \mathrm{~min}$ and $4.349 \mathrm{~min}$, and the routine blood phenotype was normal, due to the abnormal hemoglobin peak time being very close to $\mathrm{HbA2}$, so it could be easily misdiagnosed as a significant increase in $\mathrm{HbA} 2$. There are related reports of $H B A 2: c \cdot 256 \mathrm{G}>\mathrm{C}$ [23], but no related reports of HBA2:c.256G>A. The phenotype of this case was normal, the main manifestation was abnormal hemoglobin, and $\mathrm{HbA} 2$ was reduced.

Among the rare variants in the $H B B$ gene, carriers with $c$. $-100 \mathrm{G}>\mathrm{A}$, c. $-136 \mathrm{C}>\mathrm{G}, \mathrm{c} .315+5 \mathrm{G}>\mathrm{C}, \mathrm{c} .380 \mathrm{~T}>\mathrm{G}$, and c. $-81 \mathrm{~A}>\mathrm{C}$ were manifested as silent or mild $\beta$-thalassemia. The normal hematological phenotype of some cases is consistent with related reports [24-27]. Carrier with c.91A>G was manifested as mild $\beta$ thalassemia, which is consistent with related reports [28]. Carriers with c.170G $>A, c .431 \mathrm{~A}>\mathrm{G}, \mathrm{c} .232 \mathrm{C}>\mathrm{T}, \mathrm{c} .341 \mathrm{~T}>\mathrm{A}$, and c.431A $>\mathrm{G} \mathrm{s}$ had normal hematological phenotypes. The content of $\mathrm{HbA} 2$ and $\mathrm{HbF}$ was within the reference range, and abnormal hemoglobin was detected [29-32]. In the first report of c.341T>A/c.315+5G $>C$ case, abnormal hemoglobin accounted for $93.5 \%$, and $\mathrm{HbA}$ was not detected. Carrier with c.431A $>\mathrm{G}$ had the peak time of abnormal hemoglobin and $\mathrm{HbA} 2$ overlapped, and the content of each component could not be detected correctly. The blood routine examination of c. $-248 \mathrm{~A}>\mathrm{G}$ carrier was normal, mainly manifested as a decrease in $\mathrm{HbA} 2$ content [33].The phenotype of c.316-45G >C, c.316-179A >C, and c.315+308delA combined with other types of a-thalassemia may be as silent or mild $a$ thalassemia.

\section{CONCLUSIONS}

In summary, in this region, the incidences of rare gene variants and abnormal hemoglobin cases were high. SMRT technology used in the genetic diagnosis of thalassemia had wide detection spectrum with improved efficiency and accuracy over conventional methods. The application of this technology has greatly enriched the thalassemia gene mutation bank and hemoglobin gene profiles in the region, and provided a reference for better prevention and control of thalassemia. However, the pathogenicity of many rare mutant genes is still unclear, and family analysis is required, which brings great challenges to clinical genetic counseling.

\section{DATA AVAILABILITY}

Individual participant data describing the results reported in this article, after deidentification (text, tables, figures, and appendices), together with the study protocol, will be available, beginning 9 months and ending 36 months following article publication. Data will be available for investigators whose proposed use of the data has been approved by an independent review committee identified for this purpose and for individual participant data meta-analysis.

\section{REFERENCES}

1. Weatherall DJ. The evolving spectrum of the epidemiology of thalassemia. Hematol Oncol Clin North Am. 2018;32:165-75.

2. Shang $X$, Peng $Z, Y e Y$, Asan, Zhang $X$, Chen $Y$, et al. Rapid targeted nextgeneration sequencing platform for molecular screening and clinical genotyping in subjects with hemoglobinopathies. EBioMedicine. 2017;23:150-9.

3. Zebisch A, Schulz E, Grosso M, Lombardo B, Acierno G, Sill H, et al. Identification of a novel variant of epsilon-gamma-delta-beta thalassemia highlights limitations of next generation sequencing. Am J Hematol. 2015;90:E52-4.

4. Zhang $\mathrm{H}$, Li C, Li J, Hou S, Chen D, Yan H, et al. Next-generation sequencing improves molecular epidemiological characterization of thalassemia in Chenzhou Region, P.R. China. J Clin Lab Anal. 2019;33:e22845.

5. Xu L, Mao A, Liu H, Gui B, Choy KW, Huang H, et al. Long-molecule sequencing: a new approach for identification of clinically significant DNA variants in a-thalassemia and $\beta$-thalassemia carriers. J Mol Diagn. 2020;22:1087-95.

6. Liang Q, Gu W, Chen P, Li Y, Liu Y, Tian M, et al. A more universal approach to comprehensive analysis of thalassemia alleles (CATSA). J Mol Diagn. 2021;23: 1195-204.

7. Wenger AM, Peluso P, Rowell WJ, Chang PC, Hall RJ, Concepcion GT, et al. Accurate circular consensus long-read sequencing improves variant detection and assembly of a human genome. Nat Biotechnol. 2019;37:1155-62.

8. Eng B, Walsh R, Walker L, Patterson M, Waye JS. Characterization of a rare single alpha-globin gene deletion in a Chinese woman with $\mathrm{Hb} \mathrm{H}$ disease. Hemoglobin 2005;29:297-9.

9. Luo S, Chen X, Zhong Q, Wang Q, Xu Z, Qin L, et al. Analysis of rare thalassemia caused by HS-40 regulatory site deletion. Hematology. 2020;25:286-91.

10. Wang W, Ma ES, Chan AY, Prior J, Erber WN, Chan LC, et al. Single-tube multiplexPCR screen for anti-3.7 and anti-4.2 alpha-globin gene triplications. Clin Chem. 2003;49:1679-82.

11. Farashi S, Bayat N, Faramarzi Garous N, Ashki M, Montajabi Niat M, Vakili S, et al. Interaction of an a-globin gene triplication with $\beta$-globin gene mutations in Iranian patients with $\beta$-thalassemia intermedia. Hemoglobin. 2015;39:201-6.

12. Zhang $M$, Huang $H$, Chen $M$, Chen L, Wang $Y$, Lin $N$, et al. [Variant analysis for patients from Fujian area with Hong Kong aa type thalassemia]. Zhonghua Yi Xue Yi Chuan Xue Za Zhi. 2019;36:297-300.

13. Wang WC, Carter H, Choitz HC, Hall R, Hine TK, Jue DL. et al. Characterization of $\mathrm{Hb}$ volga [beta 27(B9)Ala->Asp] and $\mathrm{Hb}$ J-Wenchang-Wuming [alpha 11(A9)Lys$>$ Gln] in the population of the United States. Hemoglobin. 1993;17:67-71.

14. Lin $M$, Han ZJ, Wang Q, Zheng L, Wang Y, Yang H, et al. Molecular epidemiological survey of hemoglobinopathies in the Wuxi region of Jiangsu Province, eastern China. Hemoglobin 2013;37:454-66.

15. Henderson SJ, Timbs AT, McCarthy J, Gallienne AE, Proven M, Rugless MJ, et al. Ten years of routine $\alpha-$ and $\beta$-globin gene sequencing in UK hemoglobinopathy referrals reveals 60 novel mutations. Hemoglobin 2016;40:75-84.

16. Como PF, Barber S, Sage RE, Trent RJ, Kronenberg H. Hemoglobin Woodville: alpha (2)6(A4) aspartic acid-tyrosine. Hemoglobin 1986;10:135-41.

17. Griffiths KD, Lang A, Lehmann H, Mann JR, Plowman D, Raine DN. Haemoglobin Handsworth alpha 18 (A16) glycine leads to arginne. FEBS Lett. 1977;75:93-5.

18. Viprakasit V, Chinchang W, Glomglao W, Tanphaichitr VS. A rare association of alpha O-thalassemia (-SEA) and an initiation codon mutation (ATG->A-G) of the alpha2 gene causes $\mathrm{Hb} \mathrm{H}$ disease in Thailand. Hemoglobin 2005;29:235-40.

19. Pirastu M, Saglio G, Chang JC, Cao A, Kan YW. Initiation codon mutation as a cause of alpha thalassemia. J Biol Chem. 1984;259:12315-7.

20. Yang Y, Li DZ, He P. Hb Dapu (HBA2: C.52G>T): a novel nondeletional a-thalassemia mutation. Hemoglobin 2016;40:264-6.

21. Chang JG, Shih MC, Liu SC, Chen CM, Chan WL, Peng CT. Hb G-Chinese: a G->C substitution at codon 30 of the alpha2-globin gene creates a Pstl cutting site. Hemoglobin 2002;26:95-7.

22. Qadah T, Finlayson J, Dennis M, Ghassemifar R. Molecular and cellular analysis of three novel alpha2-globin gene promoter mutations [HBA2: C. $-59 \mathrm{C}>\mathrm{T}$ ], [HBA2: $\mathrm{C}$. $-81 \mathrm{C}>\mathrm{A}$ ] and [HBA2: c. $-91 \mathrm{G}>\mathrm{A}$ ] reveal varying patterns of transcriptional and translational activities. Pathology 2014;46:46-52.

23. Cohen-Solal M, Manesse B, Thillet J, Rosa J. Haemoglobin G Norfolk alpha 85 (F6) Asp leads to Asn. Structural characterization by sequenator analysis and functional properties of a new variant with high oxygen affinity. FEBS Lett. 1975; 50:163-7.

24. Li DZ, Liao C, Xie XM, Zhou JY. A novel mutation of $-50(\mathrm{G}->A)$ in the direct repeat element of the beta-globin gene identified in a patient with severe betathalassemia. Ann Hematol. 2009;88:1149-50.

25. Moassas F, Alabloog A, Murad H. Description of a Rare $\beta$-Globin Gene Mutation: $-86(\mathrm{C}>\mathrm{G})$ (HBB: C. $-136 \mathrm{C}>\mathrm{G})$ Observed in a Syrian Family. Hemoglobin 2018; 42:203-5.

26. Jiang NH, Liang S, Su C, Nechtman JF, Stoming TA.A novel beta-thalassemia mutation [IVS-II-5 (G->C)] in a Chinese family from Guangxi Province, P.R. China. Hemoglobin. 1993;17:563-7. 
27. Bardakdjian-Michau J, Fucharoen S, Delanoe-Garin J, Kister J, Lacombe C, Winichagoon P, et al. Hemoglobin Dhonburi alpha 2 beta $2126(\mathrm{H} 4)$ Val-Gly: a new unstable beta variant producing a beta-thalassemia intermedia phenotype in association with beta zero-thalassemia. Am J Hematol. 1990;35:96-9.

28. Waye JS, Eng B, Patterson M, Chui DH, Fernandes BJ. Novel beta-thalassemia mutation in patients of Jewish descent: [beta 30(B12)Arg->Gly or IVS-I(-2)(A$>$ G)]. Hemoglobin. 1998;22:83-5.

29. Chang JG, Shih MC, Liu SC, Chen CM, Chan WL, Lee TP. et al. Hb G-Honolulu [alpha30(B11)Glu->Gln (alpha2)], Hb J-Meinung [beta56(D7)Gly->Asp], and betathalassemia [codons 41/42 (-TCTT)] in a Taiwanese family. Hemoglobin. 2002;26: 325-8.

30. Zhao YL, Lin QF, He XW, Li YQ, Liang L. Hb Hezhou [ $[\beta 64(E 8) G l y \rightarrow S e r ; H B B$ : c.193G>A]: a novel variant on the $\beta$-globin gene. Hemoglobin 2021;45:133-5.

31. Moo-Penn WF, Hine TK, Johnson MH, Jue DL, Piersma $H$, Therrell B,Jr. et al. $\mathrm{Hb}$ Luxembourg [alpha 24(B5)Tyr-His], $\mathrm{Hb}$ Maputo [beta 47(CD6)Asp-Tyr], and $\mathrm{Hb}$ Fukuyama [beta 77(EF1)His-Tyr]. Hemoglobin. 1991;15:97-101.

32. Viprakasit V, Tachavanich $K$, Suwantol L, Pung-Amritt $P$, Chinchang W, Tanphaichitr VS. Allele related mutation specific-polymerase chain reaction for rapid diagnosis of $\mathrm{Hb}$ New York (beta 113 (G15) Val->Glu, beta(CD113 GTG->GAG). J Med Assoc Thai. 2002;85:S558-S563.

33. Tentori L, Carta SORCINI M, Buccella C. Hemoglobin Abruzzo: beta 143 (H 21) His leads to Arg. Clin Chim Acta. 1972;38:258-62.

\section{ACKNOWLEDGEMENTS}

We are grateful to our current laboratory members for their helpful comments on the manuscript.

\section{AUTHOR CONTRIBUTIONS}

LS and YT designed the study, performed experimental studies, and drafted the manuscript. CX compiled the data, contributed to writing, and helped in drafting the manuscript. ZQ contributed to revising the manuscript, $M A$ and $X R$ performed experimental studies and data analysis, ZD, YT, and TN performed analysis and provided valuable suggestions and clinical outputs. All authors read and approved the final manuscript.

\section{FUNDING}

This work was supported by the Scientific Research Fund of the Health and Family Planning Commission of Guangxi Zhuang Autonomous Region (grant no. Z20170528), the Liuzhou Science Technology Innovation Capability and Conditions Construction Project (grant no. 2018AF10501), the Key Research and Development Program of Guangxi (grant no. Guike AB18126056), the Key Research and
Development Program of Liuzhou (grant no. 2018BJ10301), and the Scientific Research and Technology Development Project of Liuzhou (no. 2018DB20501).

\section{Declarations}

\section{CONFLICT OF INTEREST}

The authors declare no competing interests.

\section{ETHICAL APPROVAL}

This study was conducted in accordance with ethical principles.

\section{INFORMED CONSENT}

All participants provided written informed consent before participating in any study procedures. Written informed consent has been obtained from the patients to publish this paper.

\section{ADDITIONAL INFORMATION}

Correspondence and requests for materials should be addressed to Tizhen Yan.

Reprints and permission information is available at http://www.nature.com/ reprints

Publisher's note Springer Nature remains neutral with regard to jurisdictional claims in published maps and institutional affiliations.

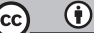

Open Access This article is licensed under a Creative Commons Attribution 4.0 International License, which permits use, sharing, adaptation, distribution and reproduction in any medium or format, as long as you give appropriate credit to the original author(s) and the source, provide a link to the Creative Commons license, and indicate if changes were made. The images or other third party material in this article are included in the article's Creative Commons license, unless indicated otherwise in a credit line to the material. If material is not included in the article's Creative Commons license and your intended use is not permitted by statutory regulation or exceeds the permitted use, you will need to obtain permission directly from the copyright holder. To view a copy of this license, visit http://creativecommons. org/licenses/by/4.0/.

(c) The Author(s) 2021 